\title{
A Novel Intelligent Technique of Invariant Statistical Embedding and Averaging via Pivotal Quantities for Optimization or Improvement of Statistical Decision Rules under Parametric Uncertainty
}

\author{
NICHOLAS A. NECHVAL, GUNDARS BERZINS, KONSTANTIN N. NECHVAL \\ Department of Mathematics \\ BVEF Research Institute, University of Latvia \\ Raina Blvd 19, Riga LV-1050 \\ LATVIA
}

\begin{abstract}
In the present paper, for intelligent constructing efficient (optimal, uniformly non-dominated, unbiased, improved) statistical decisions under parametric uncertainty, a new technique of invariant embedding of sample statistics in a decision criterion and averaging this criterion over pivots' probability distributions is proposed. This technique represents a simple and computationally attractive statistical method based on the constructive use of the invariance principle in mathematical statistics. Unlike the Bayesian approach, the technique of invariant statistical embedding and averaging via pivotal quantities (ISE\&APQ) is independent of the choice of priors and represents a novelty in the theory of statistical decisions. It allows one to eliminate unknown parameters from the problem and to find the efficient statistical decision rules, which often have smaller risk than any of the well-known decision rules. The aim of the present paper is to show how the technique of ISE\&APQ may be employed in the particular case of optimization, estimation, or improvement of statistical decisions under parametric uncertainty. To illustrate the proposed technique of ISE\&APQ, illustrative examples of intelligent constructing exact statistical tolerance limits for prediction of future outcomes coming from log-location-scale distributions under parametric uncertainty are given.
\end{abstract}

Key-Words: - Log-location-scale-distributions, Parametric uncertainty, Decision criterion, Invariant statistical embedding, Pivotal quantities, Pivot's probability distribution, Averaging, Elimination of unknown parameters, Constructing efficient statistical decision rules, Future outcomes, Exact statistical tolerance limits.

Received: October 22, 2019. Revised: February 1, 2020. Accepted: February 15, 2020. Published: February 28, 2020.

\section{Introduction}

In the present paper we consider the case, where it is known that the true distribution function belongs to a parametric family of distributions. It will be noted that, in this case, most stochastic models to solve the problems of control and optimization of system and processes are developed in the extensive literature under the assumptions that the parameter values of the underlying distributions are known with certainty. In actual practice, such is simply not the case. When these models are applied to solve real-world problems, the parameters are estimated and then treated as if they were the true values. The risk associated with using estimates rather than the true parameters is called estimation risk and is often ignored. When data are limited and (or) unreliable, estimation risk may be significant, and failure to incorporate it into the model design may lead to serious errors. Its explicit consideration is important since decision rules that are optimal in the absence of uncertainty need not even be approximately optimal in the presence of such uncertainty. The problem of determining an optimal decision rule in the absence of complete information about the underlying distribution, i.e., when we specify only the functional form of the distribution and leave some or all of its parameters unspecified, is seen to be a standard problem of statistical estimation. Unfortunately, the classical theory of statistical estimation has little to offer in general type of situation of loss function.

The bulk of the classical theory has been developed about the assumption of a quadratic, or at least symmetric and analytically simple loss structure. In some cases this assumption is made explicit, although in most it is implicit in the search for estimating procedures that have the "nice" statistical properties of unbiasedness and minimum variance. Such procedures are usually satisfactory if the estimators so generated are to be used solely for the purpose of reporting information to another party for an unknown purpose, when the loss structure is not easily discernible, or when the number of observations is large enough to support Normal approximations and asymptotic results. Unfortunately, we seldom are fortunate enough to be in asymptotic situations. Small sample sizes are 
generally the rule when estimation of system states and the small sample properties of estimators do not appear to have been thoroughly investigated. Therefore, the above procedures of the statistical estimation have long been recognized as deficient, however, when the purpose of estimation is the making of a specific decision (or sequence of decisions) on the basis of a limited amount of information in a situation where the losses are clearly asymmetric.

In this paper, we propose a new technique of invariant statistical embedding and averaging via pivotal quantities (ISE\&APQ) to solve the problems of estimation, improvement or optimization of statistical decisions under parameter uncertainty.

The technique of ISE\&APQ, the idea of which belongs to the authors, is based on the constructive use of the invariance principle in mathematical statistics and allows one to solve many problems of the theory of statistical inferences in a simple way. It allows one to yield operational, optimal information-processing rules and may be employed for finding the efficient statistical decisions for problems such as, say, multi-product newsboy problems with constraints, allocation problems of aircraft to routes under parametric uncertainty, airline set inventory control problems for multi-leg flights, etc.

The aim of the present paper is to show how the technique of ISE\&APQ may be employed in the particular case of optimization, estimation, or improvement of statistical decisions under parametric uncertainty. The technique used here is a special case of more general considerations applicable whenever the statistical problem is invariant under a group of transformations, which acts transitively on the parameter space.

\section{Technique of Invariant Statistical Embedding and Averaging via Pivotal Quantities (ISE\&APQ)}

\subsection{Preliminaries}

In the general formulation of decision theory, we observe a random sample $\mathbf{X}=\left(X_{1}, X_{2}, \ldots, X_{n}\right)$ with a sequence of iid rv's with common distribution function $F_{\theta}(x)$ where a parameter $\theta$ (in general, vector)is unknown, $\theta \in \Theta$ (parameter space). A statistic $S=S(\mathbf{X})$ is a sufficient for $\theta$ or for the family of distributions $\left\{F_{\theta}: \theta \in \boldsymbol{\Theta}\right\}$ if and only if the conditional distribution of $\mathbf{X}$, given $S=s$ does not depend on $\theta$. If we choose decision $d$ from the set of all possible decisions $\mathcal{D}$, then we suffer a loss $L(\theta, d)$. A "decision rule" is a method of choosing $d$ from $\mathcal{D}$ after observing $S$, that is, a function $d(S)=d$. Our average loss (called risk) $E_{\theta}\{L(\theta, d(S))\}$ is a function of both $\theta$ and the decision rule $d(\cdot)$, called the risk function $R(\theta, d)$, and is the criterion by which rules are compared. Thus, the expected loss (gains are negative losses) is a primary consideration in evaluating decisions. We will now define the major quantities just introduced.

Definition 1. A general statistical decision problem is a triplet $(\Theta, \mathcal{D}, L)$ and a random sample $\mathbf{X}$. The random variable $X$ from $\mathbf{X}$ (called the data) has a distribution function $F_{\theta}(x)$ where $\theta$ is unknown but it is known that $\theta \in \Theta$. $\mathbf{X}$ will denote the set of possible values of the random variable $X$. $\theta$ is called the state of nature, while the nonempty set $\Theta$ is called the parameter space. The nonempty set $\mathcal{D}$ is called the decision space or action space.

Finally, $L$ is called the loss function and to each $\theta \in \Theta$ and $d \in \mathcal{D}$ it assigns a real number $L(\theta, d)$.

Definition 2. For a statistical decision problem $(\Theta, \mathcal{D}, L), \mathbf{X}$, a (nonrandomized) decision rule is a function $d(\cdot)$ which to each $S$ assigns a member $d$ of $\mathcal{D}: d(S)=d$.

Definition 3. The risk function $R(\theta, d)$ of a decision rule $d=d(S)$ for a statistical decision problem $(\Theta, \mathcal{D}, L), \mathbf{X}$ (the expected loss or average loss when $\theta$ is the state of nature and a decision is chosen by rule $d(\cdot))$ is $R(\theta, d)=E_{\theta}\{L(\theta, d)\}$.

This paper is concerned with the implications of group theoretic structure for invariant loss functions. Our underlying structure consists of a class of probability models $(\mathcal{X}, \mathcal{A}, \mathcal{P})$, a one-one mapping $\psi$ taking $\mathcal{P}$ onto an index set $\Theta$, a measurable space of actions $(\mathcal{D}, \mathcal{B})$, and a realvalued loss function $L(\theta, d)$ defined on $\Theta \times \mathcal{D}$. We 
assume that a group $G$ of one-one $\mathcal{A}$ - measurable transformations acts on $\mathcal{X}$ and that it leaves the class of models $(\mathcal{X}, \mathcal{A}, \mathcal{P})$ invariant. We further assume that homomorphic images $\bar{G}$ and $\tilde{G}$ of $G$ act on $\Theta$ and $\mathcal{D}$, respectively. ( $\bar{G}$ may be induced on $\Theta$ through $\psi$ as in [1]; and $\tilde{G}$ may be induced on $\mathcal{D}$ through $L$, see [2]). We shall say that $L$ is invariant if for every $(\theta, d) \in \Theta \times \mathcal{D}$

$$
L(\bar{g} \theta, \tilde{g} d)=L(\theta, d), \quad g \in G .
$$

Let us assume that a loss function, $L(\theta, d)$, can be transformed as follows:

$$
L(\theta, d)=L\left(\bar{g}_{S} \theta, \tilde{g}_{s} d\right)=L^{\#}(V, \eta),
$$

where $V=V(S, \theta)$ is a pivotal quantity whose distribution does not depend on unknown parameter $\theta, \eta=\eta(S, d)$ is an invariant decision function, $S$ is a sufficient statistic for $\theta$ (or a maximum likelihood estimator of $\theta$ ). Then a risk function is given by

$$
R(\theta, d)=E_{\theta}\{L(\theta, d)\}=E\left\{L^{\#}(V, \eta)\right\},
$$

where the unknown parameter $\theta$ is eliminated from the problem. In this case, the statistical decision rule $d$ which minimizes the risk described by (3) is given by

$$
d=\eta^{*-1}(S, d),
$$

where

$$
\eta^{*}=\arg \min _{\eta} E\left\{L^{\#}(V, \eta)\right\} .
$$

Consider now, for example, the problem of estimating the location-scale parameter of a distribution belonging to a family generated by a continuous cumulative distribution function (cdf) $F$,

$$
\begin{gathered}
\mathcal{P}=\left\{P_{\theta}: F_{\theta}(x)=F\left(\frac{x-\mu}{\sigma}\right), x \in R, \theta \in \boldsymbol{\Theta}\right\}, \\
\boldsymbol{\Theta}=\{(\mu, \sigma): \mu, \sigma \in R, \sigma>0\}=\mathcal{D} .
\end{gathered}
$$

The group $G$ of location and scale changes leaves the class of models invariant. Since $\bar{G}$ induced on $\Theta$ by $P_{\theta} \rightarrow \theta$ is uniquely transitive, we may consider invariant loss function of the form

$$
L(\theta, d)=L\left(\frac{d_{1}-\mu}{\sigma}, \frac{d_{2}}{\sigma}\right),
$$

where $\theta=(\mu, \sigma)$ and $d=\left(d_{1}, d_{2}\right)$. Let us assume that there is the maximum likelihood estimator $\hat{\theta}=(\hat{\mu}, \hat{\sigma})$ of $\theta=(\mu, \sigma)$. Then a loss function, $L(\theta, d)$, can be transformed as follows:

$$
\begin{aligned}
L(\theta, d)= & L\left(\bar{g}_{\hat{\theta}} \theta, \tilde{g}_{\hat{\theta}} d\right)=L\left(\frac{d_{1}-\hat{\mu}}{\hat{\sigma}} \frac{\hat{\sigma}}{\sigma}+\frac{\widehat{\mu}-\mu}{\sigma}, \frac{d_{2}}{\hat{\sigma}} \frac{\hat{\sigma}}{\sigma}\right) \\
& =L\left(\eta_{1} V_{2}+V_{1}, \eta_{2} V_{2}\right)=L^{\#}(V, \eta),
\end{aligned}
$$

where

$$
V=\left(V_{1}=\frac{\widehat{\mu}-\mu}{\sigma}, V_{2}=\frac{\hat{\sigma}}{\sigma}\right),
$$

$V_{1}, V_{2}$ are pivotal quantities,

$$
\eta=\left(\eta_{1}=\frac{d_{1}-\hat{\mu}}{\widehat{\sigma}}, \eta_{2}=\frac{d_{2}}{\widehat{\sigma}}\right),
$$

$\eta_{1}, \eta_{2}$ are invariant decision functions. Then a risk function is given by

$$
R(\theta, d)=E_{\theta}\{L(\theta, d)\}=E\left\{L^{\#}(V, \eta)\right\},
$$

where the unknown parametric vector $\theta=(\mu, \sigma)$ is eliminated from the problem. In this case, the statistical decision rules $d_{1}, d_{2}$, which minimize the risk described by (11), are given by

$$
\begin{gathered}
d_{1}=\eta_{1}^{*-1}\left(\hat{\mu}, \widehat{\sigma}, d_{1}\right)=\widehat{\mu}+\eta_{1}^{*} \hat{\sigma}, \\
d_{2}=\eta_{2}^{*-1}\left(\widehat{\sigma}, d_{1}\right)=\eta_{2}^{*} \hat{\sigma},
\end{gathered}
$$

where

$$
\left(\eta_{1}^{*}, \eta_{2}^{*}\right)=\eta^{*}=\arg \min _{\eta} E\left\{L^{\#}(V, \eta)\right\} .
$$




\subsection{Technique of ISE\&APQ}

The technique of ISE\&APQ includes the following 3 stages:

Stage 1 (Invariant statistical embedding). At this stage, an invariant embedding of a sample statistic in the decision criterion (performance index) is carried out to construct a pivotal quantity (or simply a pivot) in order to isolate the unknown parameter from the problem (since the pivot's probability distribution does not depend on the unknown parameter).

Stage 2 (Averaging via pivotal quantities). At this stage, the decision criterion is averaged over the pivots' probability distributions in order to eliminate the unknown parameters from the problem.

Stage 3 (Decision making process). At this stage, when the unknown parameters have been eliminated from the decision criterion, it can be found an effective statistical decision rule.

\subsection{Comparison of Statistical Decision Rules}

In order to judge which statistical decision rule

might be preferred for a given situation, a comparison based on some "closeness to the true value" criteria should be made. The following approach is commonly used.

Consider two estimators, say, $d_{1}$ and $d_{2}$ having risk function $R\left(\theta, d_{1}\right)$ and $R\left(\theta, d_{2}\right)$, respectively. Then the relative efficiency of $d_{1}$ relative to $d_{2}$ is given by

$$
\text { rel.eff. }{ }_{R}\left\{d_{1}, d_{2} \mid \theta\right\}=R\left(\theta, d_{2}\right) / R\left(\theta, d_{1}\right) .
$$

When rel.eff. ${ }_{R}\left\{d_{1}, d_{2} \mid \theta_{0}\right\}<1$ for some $\theta_{0}$, we say that $d_{2}$ is more efficient than $d_{1}$ at $\theta_{0}$. If rel.eff $_{\cdot_{R}}\left\{d_{1}, d_{2} \mid \theta\right\} \leq 1$ for all $\theta$ with a strict inequality for some $\theta_{0}$, then $d_{1}$ is inadmissible relative to $d_{2}$.

\subsection{Illustrative Example}

Consider, for example, the problem of estimating a quantile $q$ of an exponential distribution on the basis of a random sample $X_{1}, \ldots, X_{n}$ of size $n \geq 2$. The exponential distribution is often used for length of life data. The exponential probability density function (pdf) is given by

$$
f_{\theta}(x)=\frac{1}{\theta} \exp \left(-\frac{x}{\theta}\right), \quad x>0, \quad \theta>0 .
$$

The cumulative distribution function (cdf) is given by

$$
F_{\theta}(x)=1-\exp \left(-\frac{x}{\theta}\right), \quad x>0, \quad \theta>0 .
$$

Quantile estimation, particularly for the exponential distribution, is important in reliability theory, life testing, and so on. Also, in statistical decision theory it is of interest to find out if the best equivariant estimator or the maximum likelihood estimator (MLE) of quantile is admissible.

Thus, the problem is to estimate the $p^{\text {th }}$ quantile $q=\vartheta \theta$ of the exponential distribution, where $0<\vartheta$ $=-\ln (1-p) ; 0<p<1$. The loss function is taken as

$$
L(\theta, d)=\left(F_{\theta}(d)-p\right)^{2},
$$

where $d$ is an estimator (decision rule) for

estimating the quantile $q$. We evaluate the performance of an estimator for quantile with the help of the risk function (decision criterion).

$$
R(\theta, d)=E_{\theta}\{L(\theta, d)\} .
$$

Assuming that the parameter $\theta$ is unknown, we find the maximum likelihood estimator (MLE) of $\theta$ given by

$$
\hat{\theta}=\sum_{i=1}^{n} X_{i} / n
$$

It is known that

$$
\widehat{\theta} \sim \varphi_{\theta}(\hat{\theta})=\frac{n^{n}}{\Gamma(n) \theta^{n}} \widehat{\theta}^{n-1} \exp \left(-\frac{n \hat{\theta}}{\theta}\right),
$$

$$
\widehat{\theta}>0, \quad \theta>0,
$$


where

$$
V=\hat{\theta} / \theta
$$

represents a pivotal quantity with the probability density function

$$
\varphi(v)=\frac{n^{n}}{\Gamma(n)} v^{n-1} \exp (-n v), \quad v>0 .
$$

To solve the above problem, the technique of invariant statistical embedding and averaging via pivotal quantities (ISE\&APQ), proposed in this paper, can be used.

Using the technique of ISE\&PQA, we have the following:

Stage 1. Invariant embedding of the MLE $\hat{\theta}$ in the decision criterion to construct the pivotal quantity $V$ :

$$
\begin{gathered}
R(\theta, d)=E_{\theta}\{L(\theta, d)\}=E_{\theta}\left\{\left(F_{\theta}(d)-p\right)^{2}\right\} \\
=E_{\theta}\left\{\left(1-p-\exp \left(-\frac{d}{\theta}\right)\right)^{2}\right\} \\
=E_{\theta}\left\{(1-p)^{2}-2(1-p) \exp \left(-\frac{d}{\theta}\right)+\exp \left(-\frac{2 d}{\theta}\right)\right\} \\
=E_{\theta}\left\{(1-p)^{2}-2(1-p) \exp \left(-\frac{d}{\hat{\theta}} \frac{\hat{\theta}}{\theta}\right)+\exp \left(-\frac{2 d}{\hat{\theta}} \frac{\widehat{\theta}}{\theta}\right)\right\} \\
=E\left\{(1-p)^{2}-2(1-p) \exp (-\eta V)+\exp (-2 \eta V)\right\},(24)
\end{gathered}
$$

where

$$
\eta=d / \hat{\theta}
$$

Stage 2. Averaging of the decision criterion over the pivot's probability distribution (23) of V:

$$
\begin{aligned}
& R(\theta, d)=E\left\{(1-p)^{2}-2(1-p) \exp (-\eta V)+\exp (-2 \eta V)\right\} \\
& =\int_{0}^{\infty}\left[(1-p)^{2}-2(1-p) \exp (-\eta v)+\exp (-2 \eta v)\right] \varphi(v) d v
\end{aligned}
$$

$$
\begin{gathered}
=\int_{0}^{\infty}\left[(1-p)^{2}-2(1-p) \exp (-\eta v)+\exp (-2 \eta v)\right] \\
\quad \times \frac{n^{n}}{\Gamma(n)} v^{n-1} \exp (-n v) d v \\
=(1-p)^{2}-2(1-p) \frac{n^{n}}{(n+\eta)^{n}}+\frac{n^{n}}{(n+2 \eta)^{n}} \\
=(1-p)^{2}-2(1-p) \frac{1}{(1+\eta / n)^{n}}+\frac{1}{(1+2 \eta / n)^{n}} .
\end{gathered}
$$

Stage 3. Process of finding the optimal statistical decision rule:

If $p=0.8, n=2$ and $\hat{\theta}=10$, it can be shown that

$$
\eta^{*}=\arg \min _{d} R(\theta, d)
$$

$$
\begin{gathered}
=\arg \min _{\eta}\left((1-p)^{2}-2(1-p) \frac{1}{(1+\eta / n)^{n}}+\frac{1}{(1+2 \eta / n)^{n}}\right) \\
=4.89598 .
\end{gathered}
$$

The optimal estimator (statistical decision rule) $d$ for estimating the quantile $q$ is given by

$$
d^{*}=\eta^{*} \hat{\theta}=4.89598 \times 10=48.9598,
$$

and the risk function is equal to

$$
R\left(\theta, d^{*}\right)=E_{\theta}\left\{L\left(\theta, d^{*}\right)\right\}=0.035121 .
$$

For comparison, the maximum likelihood estimator $d_{\mathrm{ML}}$ for estimating the quantile $q$ is given by

$$
\begin{gathered}
d_{\mathrm{ML}}=\vartheta \widehat{\theta}=-\ln (1-p) \widehat{\theta} \\
=1.609438 \times 10=16.09438,
\end{gathered}
$$

and the risk function is equal to

$$
R\left(\theta, d_{\mathrm{ML}}\right)=E_{\theta}\left\{L\left(\theta, d_{\mathrm{ML}}\right)\right\}=0.064049 .
$$

The relative efficiency of $d_{\mathrm{ML}}$ relative to $d^{*}$ is given by

$$
\text { rel.eff } \cdot_{R}\left\{d_{\mathrm{ML}}, d^{*} \mid \theta\right\}=\frac{R\left(\theta, d^{*}\right)}{R\left(\theta, d_{\mathrm{ML}}\right)}
$$




$$
=\frac{0.035121}{0.064049}=0.548346
$$

Thus, in this case, the use of $d^{*}$ leads to a reduction in the risk of about $45.2 \%$ as compared with $d_{\mathrm{ML}}$.

\section{Prediction of Future Random Quantities}

Prediction of future random quantities (future outcomes, order statistics, etc.) based on the past and current data is the most prevalent form of statistical inference. Predictive inferences for future random quantities are widely used in risk management, finance, insurance, economics, hydrology, material sciences, telecommunications, and many other industries. Predictive inferences (predictive distributions, prediction and tolerance limits) for future random quantities on the basis of the past and present knowledge represent a fundamental problem of statistics, arising in many contexts and producing varied solutions.

Statistical prediction is the process by which values for unknown observables (potential observations yet to be made or past ones which are no longer available) are inferred based on current observations and other information at hand. Whereas statistical estimation is concerned about how to get information on a distribution, usually its parameters, percentiles, distribution function, cumulative distribution function or complementary cumulative distribution function, the aim of prediction is to make inference on the value of some statistic of a sample and to enclose it within prediction limits.

\subsection{Types of Prediction}

There are the following types of prediction:

A. New-sample prediction. In this case, the data from a past sample of size $n$ are used to make prediction on one or more future units in a second sample of size $m$ from the same process or population. For example, based on previous (possibly censored) life test data, one could be interested in predicting the following:
(1) time to failure of a new item $(m=1)$;

(2) time until the $k$ th failure in a future sample of $m$ units, $m \geq k$;

(3) number of failures by time $\tau^{\bullet}$ in a future sample of $m$ units.

B. Within-sample prediction. In this case, the problem is to predict future events in a sample or process based on the early-failure data from that sample or process. For example, if $n$ units are followed until censoring time $\tau_{\mathrm{c}}$ and there are $r$ observed ordered failure times, $X_{1} \leq \ldots \leq X_{r}$, one could be interested in predicting the following:

(1) time of next failure;

(2) time until $l$ additional failures, $l \leq n-r$;

(3) number of additional failures in a future interval $\left(\tau_{c}, \tau^{\circ}\right)$.

C. New-within-sample prediction. In this case, the problem is to predict future events in a sample or process based on both the early-failure data from that sample or process and the data from a previous sample (i.e., when for predicting a future failure time of a unit in a new sample there are available both the early-failure data from that sample and the data from a previous sample).

In this paper, the new-sample prediction is considered.

\subsection{Types of Tolerance Limits}

In this paper, two types of statistical limits are defined: i) statistical $\gamma$-content tolerance limits with expected $(1-\alpha)$-confidence on future outcomes, ii) statistical tolerance limits with expected $(1-\alpha)$ confidence on future outcomes (or simply $(1-\alpha)$ prediction limit). To be specific, let $\gamma$ denote a proportion between 0 and 1 . Then one-sided statistical $\gamma$-content tolerance limit with expected $(1-\alpha)$-confidence is determined to capture a proportion $\gamma$ or more of the population, with a given expected confidence level $1-\alpha$. For example, an upper statistical $\gamma$-content tolerance limit with expected $(1-\alpha)$-confidence on future outcomes 
from a univariate population is such that with the given expected confidence level $1-\alpha$, a specified proportion $\gamma$ or more of the population will fall below the limit. A lower statistical $\gamma$-content tolerance limit with expected $(1-\alpha)$-confidence satisfies similar conditions. An upper statistical tolerance limit with expected $(1-\alpha)$-confidence is determined so that the expected proportion of the population failing below the limit is $(1-\alpha)$. A lower statistical prediction limit with expected $(1-\alpha)$ confidence satisfies similar conditions. The statistical $\gamma$-content tolerance limit with expected $(1-\alpha)$-confidence seems to be more useful than the statistical tolerance limit with expected $(1-\alpha)$ confidence but is relatively difficult to construct.

\subsection{Background}

The logical purpose for a tolerance (or prediction) limit must be the prediction of future outcomes for some (say, stochastic) process. Tolerance (prediction) limits enjoy a fairly rich history in the scientific literature and have a very important role in engineering and manufacturing applications. Patel [3] provides a review (which was fairly comprehensive at the time of publication) of tolerance intervals (limits) for many distributions as well as a discussion of their relation with confidence intervals (limits) for percentiles. Dunsmore [4] and Guenther, Patil, and Uppuluri [5] both discuss 2-parameter exponential tolerance intervals (limits) and the estimation procedure in greater detail. Engelhardt and Bain [6] discuss how to modify the formulas when dealing with type II censored data. Guenther [7] and Hahn and Meeker [8] discuss how one-sided tolerance limits can be used to obtain approximate two-sided tolerance intervals by applying Bonferroni's inequality. In Nechval et al. [9-26], the exact statistical tolerance and prediction limits are discussed under parametric uncertainty of underlying models.

In contrast to other statistical limits commonly used for statistical inference, the $\gamma$ - content tolerance limit with expected $(1-\alpha)$ - confidence is used relatively rarely. One reason is that the theoretical concept and computational complexity of the $\gamma$ - content tolerance limits with expected $(1-\alpha)$ - confidence is significantly more difficult than that of the standard confidence and prediction limits. Thus it becomes necessary to use the innovative approaches which will allow one to construct tolerance limits on future random quantities for many populations.

\section{Focus of the Paper}

\subsection{Problem Statement}

The problem can be stated more formally as follows. Let $X_{1} \leq \ldots \leq X_{r}$ be the first $r$ ordered observations of a random variable $X$ from a sample of size $n$ from a distribution with a probability density function $f_{\theta}(x)$ (distribution function $F_{\theta}(x)$, survival function $\left.\bar{F}_{\theta}(x)=1-F_{\theta}(x)\right)$ and $S$ be any statistic (say, sufficient statistic or maximum likelihood estimator) obtained from the experimental random sample $X_{1} \leq \ldots \leq X_{r}$, and let a random variable $Y$ (in a future random sample $Y_{1}$, $\left.\ldots, Y_{m}\right)$ has the same distribution with the probability density function $f_{\theta}(y)$ (distribution function $F_{\theta}(y)$, survival function $\bar{F}_{\theta}(y)=1-F_{\theta}(y)$ ), where a parameter $\theta$ (in general, vector) is common to both distributions and it is assumed that some or all numerical values of components of the parametric vector $\theta$ are unspecified.

On the basis of the experimental random sample $X_{1} \leq \ldots \leq X_{r}$ we wish to make a prediction about a future outcome of $Y_{k}$ ( $k$ th order statistic, $1 \leq k \leq m$, in a future random sample of $m$ ordered observations $Y_{1} \leq \ldots \leq Y_{m}$ ), usually in the form of one-sided statistical tolerance limits on future outcomes of $Y_{k}$ (lower $\gamma$-content tolerance limit $L_{k}$ with expected $(1-\alpha)$-confidence and upper $\gamma$ content tolerance limit $U_{k}$ with expected $(1-\alpha)$ confidence). That is, if $L_{k}$ and $U_{k}$ are functions of $S$, then $L_{k} \equiv L_{k}(S)$ is a lower statistical $\gamma$-content tolerance limit with expected $(1-\alpha)$-confidence on future outcomes of the $k$ th order statistic $Y_{k}$ if

$$
\begin{gathered}
\quad E_{\theta}\left\{\operatorname{Pr}\left(\int_{L_{k}(S)}^{\infty} g_{\theta}\left(y_{k}\right) d y_{k} \geq \gamma\right)\right\} \\
=E_{\theta}\left\{\operatorname{Pr}\left(\bar{G}_{\theta}\left(L_{k}(S)\right) \geq \gamma\right)\right\}=1-\alpha,
\end{gathered}
$$


and $U_{k} \equiv U_{k}(S)$ is an upper statistical $\gamma$-content tolerance limit with expected $(1-\alpha)$-confidence on future outcomes of the $k$ th order statistic $Y_{k}$ if

$$
\begin{array}{r}
E_{\theta}\left\{\operatorname{Pr}\left(\int_{0}^{U_{k}(S)} g_{\theta}\left(y_{k}\right) d y_{k} \geq \gamma\right)\right\} \\
=E_{\theta}\left\{\operatorname{Pr}\left(G_{\theta}\left(U_{k}(S)\right) \geq \gamma\right)\right\}=1-\alpha,
\end{array}
$$

where

$$
g_{\theta}\left(y_{k}\right)=\frac{1}{\mathrm{~B}(k, m-k+1)}\left[F_{\theta}\left(y_{k}\right)\right]^{k-1}\left[1-F_{\theta}\left(y_{k}\right)^{m-k} f_{\theta}\left(y_{k}\right)\right.
$$

is the probability density function of the $k$ th order statistic $Y_{k}$,

$$
\begin{aligned}
G_{\theta}\left(y_{k}\right) & =\operatorname{Pr}\left(Y_{k} \leq y_{k}\right)=\sum_{i=k}^{m}\left(\begin{array}{c}
m \\
i
\end{array}\right)\left[F_{\theta}\left(y_{k}\right)\right]^{i}\left[1-F_{\theta}\left(y_{k}\right)\right]^{m-i} \\
& =\sum_{i=k}^{m}\left(\begin{array}{c}
m \\
i
\end{array}\right)\left[1-\bar{F}_{\theta}\left(y_{k}\right)\right]^{i}\left[\bar{F}_{\theta}\left(y_{k}\right)\right]^{m-i} \\
& =\sum_{i=k}^{m}\left(\begin{array}{c}
m \\
i
\end{array}\right) \sum_{j=0}^{i}\left(\begin{array}{c}
i \\
j
\end{array}\right)(-1)^{j}\left[\bar{F}_{\theta}\left(y_{k}\right]^{m-i+j}\right. \\
= & \int_{0}^{F_{\theta}\left(y_{k}\right)} \frac{1}{\mathrm{~B}(k, m-k+1)} \tau^{k-1}(1-\tau)^{m-k} d \tau
\end{aligned}
$$

is the probability distribution function of the $k$ th order statistic $Y_{k}$. It can be shown that

$$
\frac{d G_{\theta}\left(y_{k}\right)}{d y_{k}}=g_{\theta}\left(y_{k}\right)
$$

Further, $L_{k}^{\circ} \equiv L_{k}^{\circ}(S) \quad$ is a lower statistical tolerance limit with expected $(1-\alpha)$-confidence on future outcomes of the $k$ th order statistic $Y_{k}$ from a set of $m$ future ordered observations $Y_{1} \leq \ldots \leq Y_{m}$ if it satisfies

$$
\begin{gathered}
E_{\theta}\left\{\operatorname{Pr}\left(Y_{k}>L_{k}^{\circ}(S)\right)\right\}=E_{\theta}\left\{\int_{L_{k}^{\circ}(S)}^{\infty} g_{\theta}\left(y_{k}\right) d y_{k}\right\} \\
=E_{\theta}\left\{\bar{G}_{\theta}\left(L_{k}^{\circ}(S)\right)\right\}=1-\alpha .
\end{gathered}
$$

$U_{k}^{\circ} \equiv U_{k}^{\circ}(S)$ is an upper statistical tolerance limit with expected $(1-\alpha)$-confidence on future outcomes of the $k$ th order statistic $Y_{k}$ from a set of $m$ future ordered observations $Y_{1} \leq \ldots \leq Y_{m}$ if it satisfies

$$
\begin{gathered}
E_{\theta}\left\{\operatorname{Pr}\left(Y_{k} \leq U_{k}^{\circ}(S)\right)\right\}=E_{\theta}\left\{\int_{0}^{U_{k}^{\circ}(S)} g_{\theta}\left(y_{k}\right) d y_{k}\right\} \\
=E_{\theta}\left\{G_{\theta}\left(U_{k}^{\circ}(S)\right)\right\}=1-\alpha .
\end{gathered}
$$

In this paper, a new technique for intelligent constructing the statistical $\gamma$-content tolerance limits with expected $(1-\alpha)$-confidence as well as the statistical tolerance limits with expected $(1-\alpha)$ confidence on order statistics in future samples is proposed. For illustration, the extreme-value and Weibull distributions are considered.

\subsection{Extreme-Value Distribution}

This distribution is used in many research fields including, among others, life testing and water resource management. This is the so-called first asymptotic distribution of extreme values, hereafter referred to simply as the extreme-value distribution. The distribution is extensively used in a number of areas as a lifetime distribution and sometimes referred to as the Gumbel distribution, after E. J. Gumbel, who had pioneered its use (Gumbel [27]).

Let $X_{1} \leq \ldots \leq X_{r}$ be the first $r$ ordered observations of a random variable $X$ from a sample of size $n$ from an extreme-value distribution with the pdf (probability density function),

$$
f_{\theta}(x)=\frac{1}{\theta_{2}} \exp \left(\frac{x-\theta_{1}}{\theta_{2}}\right) \exp \left[-\exp \left(\frac{x-\theta_{1}}{\theta_{2}}\right)\right],-\infty<x<\infty,
$$

and cdf (cumulative distribution function),

$$
F_{\theta}(x)=1-\exp \left[-\exp \left(\frac{x-\theta_{1}}{\theta_{2}}\right)\right],-\infty<x<\infty
$$

indexed by location and scale parameters $\theta_{1}$ and $\theta_{2}$, where $\theta=\left(\theta_{1}, \theta_{2}\right)$.It is assumed that the parameters $\theta_{1}\left(-\infty<\theta_{1}<\infty\right)$ and $\theta_{2}>0$ are unknown.

In Type II censoring, which is of primary interest here, the number of survivors are fixed and 
$X_{r}$ is a random variable. The MLE's $\hat{\theta}_{1}$ and $\hat{\theta}_{2}$ of the parameters $\theta_{1}$ and $\theta_{2}$, respectively, are solutions of

$$
\begin{gathered}
\hat{\theta}_{1}=\ln \left[r^{-1}\left(\sum_{i=1}^{r} \exp \left(\frac{x_{i}}{\widehat{\theta}_{2}}\right)+(n-r) \exp \left(\frac{x_{r}}{\widehat{\theta}_{2}}\right)\right)\right]^{\hat{\theta}_{2}}, \\
\hat{\theta}_{2}=\left[\sum_{i=1}^{r} x_{i} \exp \left(\frac{x_{i}}{\hat{\theta}_{2}}\right)+(n-r) x_{r} \exp \left(\frac{x_{r}}{\hat{\theta}_{2}}\right)\right] \\
\times\left[\sum_{i=1}^{r} \exp \left(\frac{x_{i}}{\hat{\theta}_{2}}\right)+(n-r) \exp \left(\frac{x_{r}}{\hat{\theta}_{2}}\right)\right]^{-1}-\frac{1}{r} \sum_{i=1}^{r} x_{i} .
\end{gathered}
$$

In terms of the extreme-value distribution variates, we have that

$$
W_{1}=\frac{\hat{\theta}_{1}-\theta_{1}}{\theta_{2}}, \quad W_{2}=\frac{\hat{\theta}_{2}}{\theta_{2}}, \quad W_{3}=\frac{\hat{\theta}_{1}-\theta_{1}}{\hat{\theta}_{2}}
$$

are pivotal quantities. The probability density functions of the pivotal quantities do not depend on the parameters. It can be shown that the joint pdf of the pivotal quantities

$$
W_{1}=\frac{\hat{\theta}_{1}-\theta_{1}}{\theta_{2}}, \quad W_{2}=\frac{\hat{\theta}_{2}}{\theta_{2}},
$$

conditional on fixed

$$
\mathbf{S}^{(r)}=\left(S_{i}, \ldots, S_{r}\right),
$$

where

$$
S_{i}=\frac{X_{i}-\hat{\theta}_{1}}{\hat{\theta}_{2}}, \quad i=1, \ldots, r
$$

are ancillary statistics, any $r-2$ of which form a functionally independent set, $\hat{\theta}_{1}$ and $\hat{\theta}_{2}$ are the maximum likelihood estimates for $\theta_{1}$ and $\theta_{2}$, respectively, based on the first $r$ ordered observations $\left(X_{1} \leq \ldots \leq X_{r}\right)$ from a sample of size $n$ from the extreme-value distribution (8), which can be found from solution of (42) and (43), is given by

$$
f_{n}\left(w_{1}, w_{2} \mid \mathbf{s}^{(r)}\right)=\left(\frac{\left(e^{w_{1}}\right)^{r}}{\Gamma(r)}\left[\sum_{i=1}^{r} \exp \left(s_{i} w_{2}\right)+(n-r) \exp \left(s_{r} w_{2}\right)\right]^{r}\right.
$$

$$
\begin{gathered}
\left.\times \exp \left(-e^{w_{1}}\left[\sum_{i=1}^{r} \exp \left(s_{i} w_{2}\right)+(n-r) \exp \left(s_{r} w_{2}\right)\right]\right)\right) \\
\times\left(\frac{1}{v\left(\mathbf{s}^{(r)}\right)} w_{2}^{r-2} \prod_{i=1}^{r} \exp \left(s_{i} w_{2}\right)\right. \\
\left.\times\left[\sum_{i=1}^{r} \exp \left(s_{i} w_{2}\right)+(n-r) \exp \left(s_{r} w_{2}\right)\right]^{-r}\right) \\
=f_{n}\left(w_{1} \mid \mathbf{s}^{(r)}, w_{2}\right) f_{n}\left(w_{2} \mid \mathbf{s}^{(r)}\right), \\
w_{1} \in(-\infty, \infty), \quad w_{2} \in(0, \infty),
\end{gathered}
$$

where

$$
\begin{gathered}
f_{n}\left(w_{1} \mid \mathbf{s}^{(r)}, w_{2}\right)=\frac{\left(e^{w_{1}}\right)^{r}}{\Gamma(r)}\left[\sum_{i=1}^{r} \exp \left(s_{i} w_{2}\right)+(n-r) \exp \left(s_{r} w_{2}\right)\right]^{r} \\
\times \exp \left(-e^{w_{1}}\left[\sum_{i=1}^{r} \exp \left(s_{i} w_{2}\right)+(n-r) \exp \left(s_{r} w_{2}\right)\right]\right), \\
w_{1} \in(-\infty, \infty) .
\end{gathered}
$$

$$
f_{n}\left(w_{2} \mid \mathbf{s}^{(r)}\right)=\frac{1}{v\left(\mathbf{s}^{(r)}\right)} w_{2}^{r-2} \prod_{i=1}^{r} \exp \left(s_{i} w_{2}\right)
$$$$
\times\left[\sum_{i=1}^{r} \exp \left(s_{i} w_{2}\right)+(n-r) \exp \left(s_{r} w_{2}\right)\right]^{-r}, w_{2} \in(0, \infty),
$$

$$
v\left(\mathbf{s}^{(r)}\right)=\int_{0}^{\infty} w_{2}^{r-2} \prod_{i=1}^{r} \exp \left(s_{i} w_{2}\right)
$$

$$
\times\left[\sum_{i=1}^{r} \exp \left(s_{i} w_{2}\right)+(n-r) \exp \left(s_{r} w_{2}\right)\right]^{-r} d w_{2}
$$

is the normalizing constant. If a pivotal quantity is given by

$$
W=e^{W_{1}}\left[\sum_{i=1}^{r} \exp \left(S_{i} W_{2}\right)+(n-r) \exp \left(S_{r} W_{2}\right)\right],
$$

it follows from (49) that

$$
W \sim g_{r}(w)=\frac{1}{\Gamma(r)} w^{r-1} \exp (-w), \quad w \in(0, \infty) .
$$




\subsection{Two-Parameter Weibull Distribution}

The two-parameter Weibull distribution is one of the most widely used life distributions in reliability analysis. This distribution is very flexible, and can, through an appropriate choice of parameters, model many types of failure rate behaviors. It has wide applications in diverse disciplines.

Let $\underline{X}_{1} \leq \ldots \leq \underline{X}_{r}$ be the first $r$ ordered observations of a random variable $\underline{X}$ from a sample of size $n$ from a two-parameter Weibull distribution with the pdf,

$$
f_{\underline{\theta}}(\underline{x})=\frac{\delta}{\beta}(\underline{\underline{x}})^{\delta-1} \exp \left[-\left(\frac{\underline{x}}{\beta}\right)^{\delta}\right], \underline{x}>0, \beta>0, \delta>0,
$$

and cdf,

$$
F_{\underline{\theta}}(\underline{x})=1-\exp \left[-\left(\frac{\underline{x}}{\beta}\right)^{\delta}\right], \underline{x}>0, \beta>0, \delta>0,
$$

indexed by scale and shape parameters $\beta$ and $\delta$, where $\underline{\theta}=(\beta, \delta)$. It is assumed that the parameters $\beta$ and $\delta$ are unknown. This distribution is directly related to the extreme-value distribution by the easily shown fact that if $\underline{X}$ has a Weibull distribution (54), then $X=\ln \underline{X}$ has an extremevalue distribution with $\theta_{1}=\ln \beta$ and $\theta_{2}=\delta^{-1}$. In analyzing data it is often convenient to work with $\log$ times, the extreme-value distribution arises when lifetimes are taken to be Weibull distributed. The MLE's of the Weibull parameters $\beta$ and $\delta$ are $\widehat{\beta}=\exp \widehat{\theta}_{1}$ and $\widehat{\delta}=\widehat{\theta}_{2}^{-1}$. If desired, the maximum likelihood equations (42) and (43) can be written in Weibull form and solved directly from the start. The equations are

$$
\begin{gathered}
\hat{\beta}=\left(r^{-1}\left(\sum_{i=1}^{r} \underline{x}_{i}^{\hat{\delta}}+(n-r) \underline{x}_{r}^{\hat{\delta}}\right)\right)^{1 / \hat{\delta}}, \\
\widehat{\delta}=\left[\begin{array}{l}
\left(\sum_{i=1}^{r} \underline{x}_{i}^{\hat{\delta}} \ln \underline{x}_{i}+(n-r) \underline{x}_{r}^{\hat{\delta}} \ln \underline{x}_{r}\right) \\
\times\left(\sum_{i=1}^{r} \underline{x}_{i}^{\hat{\delta}}+(n-r) \underline{x}_{r}^{\hat{\delta}}\right)^{-1}-\frac{1}{r} \sum_{i=1}^{r} \ln \underline{x}_{i}
\end{array}\right]^{-1} .
\end{gathered}
$$

In terms of the Weibull variates, we have that

$$
V_{1}=\left(\frac{\widehat{\beta}}{\beta}\right)^{\delta}, \quad V_{2}=\frac{\delta}{\widehat{\delta}}, \quad V_{3}=\left(\frac{\widehat{\beta}}{\beta}\right)^{\hat{\delta}}
$$

are pivotal quantities. The probability density functions of the pivotal quantities do not depend on the parameters. It can be shown that the joint pdf of the pivotal quantities

$$
V_{1}=\left(\frac{\widehat{\beta}}{\beta}\right)^{\delta}, \quad V_{2}=\frac{\delta}{\widehat{\delta}},
$$

conditional on fixed

where

$$
\mathbf{Z}^{(r)}=\left(Z_{i}, \ldots, Z_{r}\right),
$$

$$
Z_{i}=\left(\frac{\underline{X}_{i}}{\hat{\beta}}\right)^{\hat{\delta}}, \quad i=1, \ldots, r,
$$

are ancillary statistics, any $r-2$ of which form a functionally independent set, $\widehat{\beta}$ and $\widehat{\delta}$ are the maximum likelihood estimates for $\beta$ and $\delta$, respectively, based on the first $r$ ordered observations $\underline{X}_{1} \leq \ldots \leq \underline{X}_{r}$ from a sample of size $n$ from the two-parameter Weibull distribution (54), which can be found from solution of (56) and (57), is given by

$$
\begin{gathered}
f_{n}\left(v_{1}, v_{2} \mid \mathbf{z}^{(r)}\right)=\left(\begin{array}{l}
\frac{v_{1}^{r-1}}{\Gamma(r)}\left[\sum_{i=1}^{r} z_{i}^{v_{2}}+(n-r) z_{r}^{v_{2}}\right]^{r} \\
\times \exp \left(-v_{1}\left[\sum_{i=1}^{r} z_{i}^{v_{2}}+(n-r) z_{r}^{v_{2}}\right]\right)
\end{array}\right) \\
\times\left(\frac{1}{\vartheta\left(\mathbf{z}^{(r)}\right)} v_{2}^{r-2} \prod_{i=1}^{r} z_{i}^{v_{2}}\left[\sum_{i=1}^{r} z_{i}^{v_{2}}+(n-r) z_{r}^{v_{2}}\right]^{-r}\right) \\
=f_{n}\left(v_{1} \mid \mathbf{z}^{(r)}, v_{2}\right) f_{n}\left(v_{2} \mid \mathbf{z}^{(r)}\right), \quad v_{1} \in(0, \infty), v_{2} \in(0, \infty),(62)
\end{gathered}
$$

where

$$
\begin{gathered}
f_{n}\left(v_{1} \mid \mathbf{z}^{(r)}, v_{2}\right)=\frac{1}{\Gamma(r)}\left[\sum_{i=1}^{r} z_{i}^{v_{2}}+(n-r) z_{r}^{v_{2}}\right]^{r} \\
\times v_{1}^{r-1} \exp \left(-v_{1}\left[\sum_{i=1}^{r} z_{i}^{v_{2}}+(n-r) z_{r}^{v_{2}}\right]\right), v_{1} \in(0, \infty),
\end{gathered}
$$




$$
\begin{gathered}
f_{n}\left(v_{2} \mid \mathbf{z}^{(r)}\right)=\frac{1}{\vartheta\left(\mathbf{z}^{(r)}\right)} v_{2}^{r-2} \prod_{i=1}^{r} z_{i}^{v_{2}}\left(\sum_{i=1}^{r} z_{i}^{v_{2}}+(n-r) z_{r}^{v_{2}}\right)^{-r}, \\
v_{2} \in(0, \infty) \\
\vartheta\left(\mathbf{z}^{(r)}\right)=\int_{0}^{\infty} v_{2}^{r-2} \prod_{i=1}^{r} z_{i}^{v_{2}}\left(\sum_{i=1}^{r} z_{i}^{v_{2}}+(n-r) z_{r}^{v_{2}}\right)^{-r} d v_{2}
\end{gathered}
$$

is the normalizing constant. If a pivotal quantity is given by

$$
V=V_{1}\left[\sum_{i=1}^{r} Z_{i}^{V_{2}}+(n-r) Z_{r}^{V_{2}}\right]
$$

it follows from (31) that

$$
V \sim g_{r}(v)=\frac{1}{\Gamma(r)} v^{r-1} \exp (-v), \quad v \in(0, \infty)
$$

\section{Constructing Statistical $\gamma$-Content}

\section{Tolerance Limits with Expected}

\section{$(1-\alpha)$-Confidence}

\subsection{Constructing Lower Statistical $\gamma$ - Content Tolerance Limit with Expected (1- $\alpha)$-Confidence}

Theorem 1. Let $X_{1} \leq \ldots \leq X_{r}$ be the first $r$ ordered observations of a random variable $X(=\ln \underline{X})$ from a sample of size $n$ from an extreme-value distribution defined by the probability density function (8). Then a lower statistical $\gamma$-content tolerance limit with expected $(1-\alpha)$-confidence, $L_{k}$ $\equiv L_{k}(S)$, on future outcomes of the $k$ th order statistic $Y_{k}\left(=\ln \underline{Y}_{k}\right)$ from a set of $m$ future ordered observations $Y_{1} \leq \ldots \leq Y_{m}$ also from the distribution (40), which satisfies (33) is given by

$$
L_{k}=\hat{\theta}_{1}+\hat{\theta}_{2} \ln \eta_{L_{k}}=\ln \underline{L}_{k}
$$

where $\eta_{L_{k}}$ is a tolerance factor determined by

$$
\eta_{L_{k}}
$$

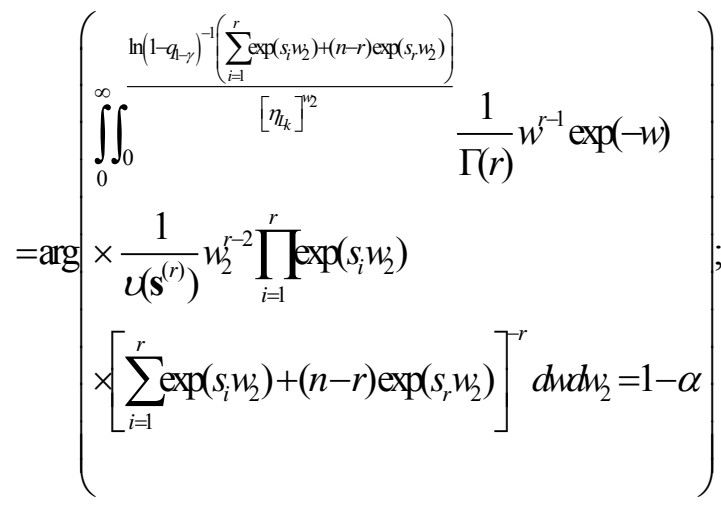

the maximum likelihood estimates $\hat{\theta}_{1}$ and $\hat{\theta}_{2}$ of the parameters $\theta_{1}$ and $\theta_{2}$ are determined from (42) and (43), respectively; the ancillary statistics $S_{i}$, $i=1, \ldots, r$, are given by (47); $q_{1-\gamma}$ is a quantile of the beta distribution satisfying

$$
\int_{0}^{a_{1-\gamma}} \frac{1}{\mathrm{~B}(k, m-k+1)} \tau^{k-1}(1-\tau)^{m-k} d \tau=1-\gamma
$$

Proof. It follows from (35) and (36) that

$$
\begin{gathered}
\operatorname{Pr}\left(\int_{L_{k}}^{\infty} g_{\theta}\left(y_{k}\right) d y_{k} \geq \gamma\right)=\operatorname{Pr}\left(\bar{G}_{\theta}\left(L_{k}\right) \geq \gamma\right)=\operatorname{Pr}\left(1-G_{\theta}\left(L_{k}\right) \geq \gamma\right) \\
=\operatorname{Pr}\left(1-\int_{0}^{F_{\theta}\left(L_{k}\right)} \frac{1}{\mathrm{~B}(k, m-k+1)} \tau^{k-1}(1-\tau)^{m-k} d \tau \geq \gamma\right) \\
=\operatorname{Pr}\left(\int_{0}^{F_{\theta}\left(L_{k}\right)} \frac{1}{\mathrm{~B}(k, m-k+1)} \tau^{k-1}(1-\tau)^{m-k} d \tau \leq 1-\gamma\right) \\
=\operatorname{Pr}\left(1-\exp \left[-\exp \left(\frac{L_{k}-\theta_{1}}{\theta_{2}}\right)\right] \leq q_{1-\gamma}\right) \\
=\operatorname{Pr}\left(\exp \left[-\exp \left(\frac{L_{k}-\theta_{1}}{\theta_{2}}\right)\right] \geq 1-q_{1-\gamma}\right) \\
=\operatorname{Pr}\left(e^{W_{1}} \leq \frac{\ln \left(1-q_{1-\gamma}\right)^{-1}}{\left[\exp \left(\left(L_{k}-\hat{\theta}_{1}\right) / \hat{\theta}_{2}\right)\right]^{W_{2}}}\right)
\end{gathered}
$$




$$
\begin{aligned}
& =\operatorname{Pr}\left(\begin{array}{l}
e^{W_{1}}\left(\sum_{i=1}^{r} \exp \left(S_{i} W_{2}\right)+(n-r) \exp \left(S_{r} W_{2}\right)\right) \\
\left.\leq \frac{\ln \left(1-q_{1-\gamma}\right)^{-1}\left(\sum_{i=1}^{r} \exp \left(S_{i} W_{2}\right)+(n-r) \exp \left(S_{r} W_{2}\right)\right)}{\left[\exp \left(\left(L_{k}-\hat{\theta}_{1}\right) / \hat{\theta}_{2}\right)\right]^{W_{2}}}\right)
\end{array}\right) \cdot(71) \\
& =\operatorname{Pr}\left(W \leq \frac{\ln \left(1-q_{1-\gamma}\right)^{-1}\left(\sum_{i=1}^{r} \exp \left(S_{i} W_{2}\right)+(n-r) \exp \left(S_{r} W_{2}\right)\right)}{\left[\exp \left(\left(L_{k}-\hat{\theta}_{1}\right) / \hat{\theta}_{2}\right)\right]^{W_{2}}}\right)
\end{aligned}
$$

Using averaging via pivotal quantity, it follows from (33) and (71) that

$$
E_{\theta}\left\{\operatorname{Pr}\left(\int_{L_{k}(S)}^{\infty} g_{\theta}\left(y_{k}\right) d y_{k} \geq \gamma\right)\right\}
$$

$$
=E\left\{\operatorname{Pr}\left\{W \leq \frac{\ln \left(1-q_{1-\gamma}\right)^{-1}\left(\sum_{i=1}^{r} \exp \left(S_{i} W_{2}\right)+(n-r) \exp \left(S_{r} W_{2}\right)\right)}{\left[\exp \left(\left(L_{k}-\hat{\theta}\right) / \hat{\theta}_{2}\right)\right]^{W_{2}}}\right)\right\}
$$

$$
=E\left\{\int_{0}^{\frac{\ln \left(1-q_{1-\gamma}\right)^{-1}\left(\sum_{i=1}^{r} \exp \left(S_{i} W_{2}\right)+(n-r) \exp \left(S_{r} W_{2}\right)\right)}{\left[\exp \left(\left(L_{k}-\hat{\theta}_{1}\right) / \hat{\theta}_{2}\right)\right]^{W_{2}}}} g_{r}(w) d w\right\}
$$

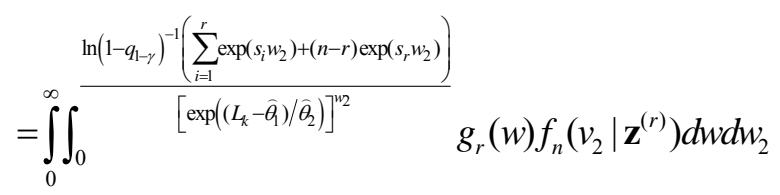

$$
\begin{gathered}
=\int_{0}^{\infty} \int_{0}^{\frac{\ln \left(1-q_{1-\gamma}\right)^{-1}\left(\sum_{i=1}^{r} \exp \left(s_{i} w_{2}\right)+(n-r) \exp \left(s_{r} w_{2}\right)\right)}{\left[\exp \left(\left(L_{k}-\hat{\theta}_{1}\right) / \hat{\theta}_{2}\right)\right]^{w_{2}}}} \frac{1}{\Gamma(r)} w^{r-1} \exp (-w) \\
\times \frac{1}{v\left(\mathbf{s}^{(r)}\right)} w_{2}^{r-2} \prod_{i=1}^{r} \exp \left(s_{i} w_{2}\right)
\end{gathered}
$$$$
\times\left[\sum_{i=1}^{r} \exp \left(s_{i} w_{2}\right)+(n-r) \exp \left(s_{r} w_{2}\right)\right]^{-r} d w d w_{2}=1-\alpha .
$$

It follows from (72) that

$$
L_{k}
$$

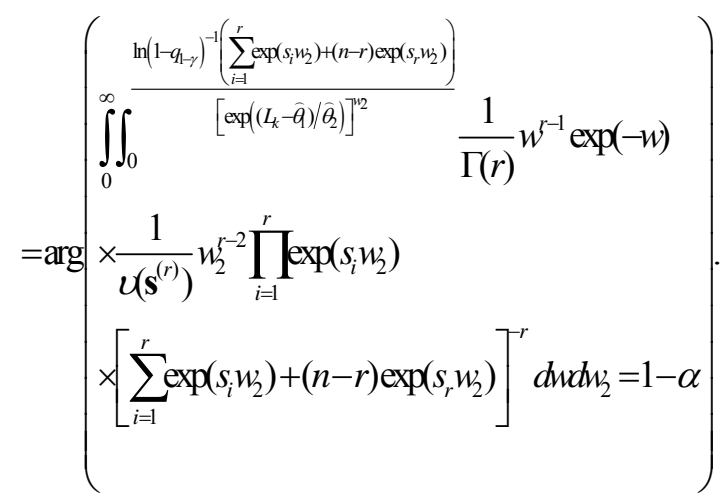

Assuming that

$$
\exp \left(\frac{L_{k}-\hat{\theta}_{1}}{\hat{\theta}_{2}}\right)=\eta_{L_{k}},
$$

we have (68). This completes the proof.

Theorem 2. Let $\underline{X}_{1} \leq \ldots \leq \underline{X}_{r}$ be the first $r$ ordered observations of a random variable $\underline{X}(=\exp X)$ from a sample of size $n$ from a twoparameter Weibull distribution defined by the probability density function (54). Then a lower statistical $\gamma$-content tolerance limit with expected (1- $\alpha)$-confidence, $\underline{L}_{k} \equiv \underline{L}_{k}(\underline{S})$, on future outcomes of the $k$ th order statistic $\underline{Y}_{k}\left(=\exp Y_{k}\right)$ from a set of $m$ future ordered observations $\underline{Y}_{1} \leq \ldots \leq \underline{Y}_{m}$ also from the distribution (54), which satisfies (33) is given by

$$
\underline{L}_{k}=\eta_{\underline{L}_{k}}^{1 / \hat{\delta}} \widehat{\beta}=\exp L_{k},
$$

where $\eta_{L_{k}}$ is a tolerance factor determined by

$$
\begin{aligned}
& \eta_{\underline{L}_{k}}
\end{aligned}
$$

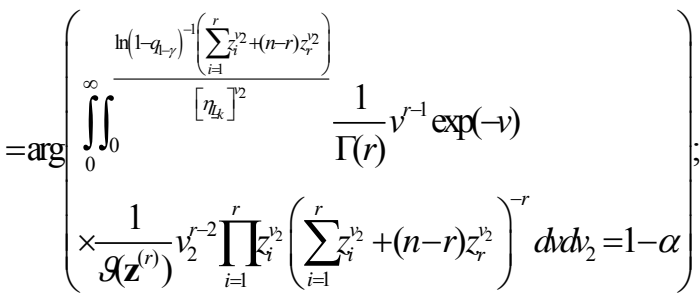

the maximum likelihood estimates $\widehat{\beta}$ and $\widehat{\delta}$ of the parameters $\beta$ and $\delta$ are determined from (56) and (57), respectively; the ancillary statistics $Z_{i}, i=1, \ldots$, $r$, are given by $(61) ; q_{1-\gamma}$ is a quantile of the beta distribution satisfying (70). 
Proof. The proof is similar to that of Theorem 1 and so it is omitted here.

Inference 1 (for the tolerance factors $\eta_{\underline{L}_{k}}$ and $\left.\eta_{L_{k}}\right)$. It follows from (74) and (75) that

$$
\eta_{\underline{L}_{k}}=\left(\frac{\underline{L}_{k}}{\hat{\beta}}\right)^{\hat{\delta}}=\left(\frac{\exp L_{k}}{\exp \hat{\theta}_{1}}\right)^{1 / \hat{\theta}_{2}}=\exp \left(\frac{L_{k}-\widehat{\theta}_{1}}{\widehat{\theta}_{2}}\right)=\eta_{L_{k}} \cdot
$$

\subsection{Constructing Upper Statistical $\gamma$ - Content Tolerance Limit with Expected $(1-\alpha)$-Confidence}

Theorem 3. Let $X_{1} \leq \ldots \leq X_{r}$ be the first $r$ ordered observations of a random variable $X(=\ln \underline{X})$ from a sample of size $n$ from an extreme-value distribution defined by the probability density function (40). Then an upper statistical $\gamma$-content tolerance limit with $(1-\alpha)$-confidence, $U_{k} \equiv U_{k}(S)$, on future outcomes of the $k$ th order statistic $Y_{k}\left(=\ln \underline{Y}_{k}\right) \quad$ from a set of $m$ future ordered observations $Y_{1} \leq \ldots \leq Y_{m}$ also from the distribution (40), which satisfies (34) is given by

$$
U_{k}=\hat{\theta}_{1}+\hat{\theta}_{2} \ln \eta_{U_{k}}=\ln \underline{U}_{k},
$$

where $\eta_{U_{k}}$ is a tolerance factor determined by

$$
\eta_{U_{k}}=\arg \left(\begin{array}{l}
\int_{0}^{\infty} \frac{\ln \left(1-q_{q^{\prime}}\right)^{-1}\left(\sum_{i=1}^{r} \exp \left(s_{i} w_{2}\right)+(n-r) \exp \left(s_{r} w_{2}\right)\right)}{\left[\eta_{U_{k}}\right]^{\gamma_{2}}} \frac{1}{\Gamma(r)} w^{r-1} \exp (-w) \\
\times \frac{1}{v\left(\mathbf{s}^{(r)}\right)} W_{2}^{r-2} \prod_{i=1}^{r} \exp \left(s_{i} w_{2}\right) \\
\times\left[\sum_{i=1}^{r} \exp \left(s_{i} w_{2}\right)+(n-r) \exp \left(s_{r} w_{2}\right)\right]^{-r} d w d w_{2}=\alpha
\end{array}\right) ;
$$

the maximum likelihood estimates $\hat{\theta}_{1}$ and $\hat{\theta}_{2}$ of the parameters $\theta_{1}$ and $\theta_{2}$ are determined from (42) and (43), respectively; the ancillary statistics $S_{i}$, $i=1, \ldots, r$, are given by (47); $q_{\gamma}$ is a quantile of the beta distribution satisfying (70).

Proof. The upper statistical $\gamma$-content tolerance limit with expected $(1-\alpha)$-confidence, $U_{k} \equiv U_{k}(S)$, is obtained from a lower statistical $\gamma$-content tolerance limit with expected $(1-\alpha)$-confidence, $L_{k} \equiv L_{k}(S)$, by replacing $\gamma$ by $1-\gamma$, and $1-\alpha$ by $\alpha$. This completes the proof.

Theorem 4. Let $\underline{X}_{1} \leq \ldots \leq \underline{X}_{r}$ be the first $r$ ordered observations of a random variable $\underline{X}(=\exp X)$ from a sample of size $n$ from a twoparameter Weibull distribution defined by the probability density function (54). Then an upper statistical $\gamma$-content tolerance limit with $(1-\alpha)$ confidence, $\underline{U}_{k} \equiv \underline{U}_{k}(\underline{S})$, on future outcomes of the $k$ th order statistic $\underline{Y}_{k}\left(=\exp Y_{k}\right)$ from a set of $m$ future ordered observations $\underline{Y}_{1} \leq \ldots \leq \underline{Y}_{m}$ also from the distribution (54), which satisfies (34) is given by

$$
\underline{U}_{k}=\eta_{\underline{U}_{k}}^{1 / \hat{\delta}} \widehat{\beta}=\exp U_{k},
$$

where $\eta_{U_{k}}$ is a tolerance factor determined by

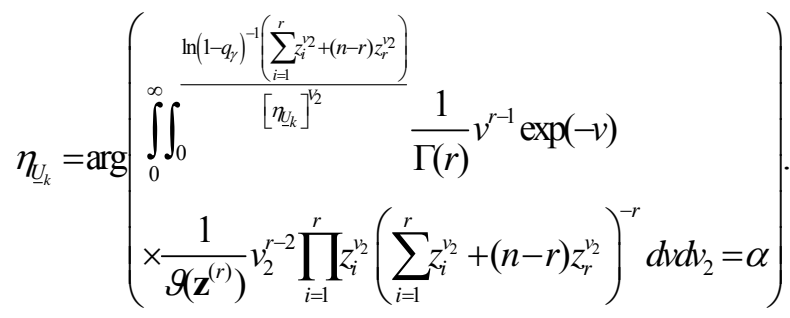

Proof. The upper statistical $\gamma$-content tolerance limit with expected (1- $\alpha)$-confidence, $\underline{U}_{k} \equiv \underline{U}_{k}(\underline{S})$, is obtained from a lower statistical $\gamma$-content tolerance limit with expected $(1-\alpha)$-confidence, $\underline{L}_{k} \equiv \underline{L}_{k}(\underline{S})$, by replacing $\gamma$ by $1-\gamma$, and $1-\alpha$ by $\alpha$. This completes the proof.

Inference 2 (for the tolerance factors $\eta_{\underline{U}_{k}}$ and $\left.\eta_{U_{k}}\right)$. It follows from (78) and (80) that

$$
\eta_{\underline{U}_{k}}=\left(\frac{\underline{U}_{k}}{\widehat{\beta}}\right)^{\hat{\delta}}=\left(\frac{\exp U_{k}}{\exp \widehat{\theta}_{1}}\right)^{1 / \hat{\theta}_{2}}=\exp \left(\frac{U_{k}-\widehat{\theta}_{1}}{\widehat{\theta}_{2}}\right)=\eta_{U_{k}} .
$$




\section{Constructing Statistical Tolerance Limits with Expected (1- $\alpha)$ - Confidence}

\subsection{Constructing Lower Statistical}

Tolerance Limit with Expected (1- $\alpha)$ -

\section{Confidence}

Theorem 5. Let $X_{1} \leq \ldots \leq X_{r}$ be the first $r$ ordered observations of a random variable $X(=\ln \underline{X})$ from a sample of size $n$ from an extreme-value distribution defined by the probability density function (40). Then a lower statistical tolerance limit with expected $(1-\alpha)$-confidence, $L_{k}^{\circ} \equiv L_{k}^{\circ}(S)$, on future outcomes of the $k$ th order statistic $Y_{k}\left(=\ln \underline{Y}_{k}\right)$ from a set of $m$ future ordered observations $Y_{1} \leq \ldots \leq Y_{m}$ also from the distribution (40), which satisfies (38) is given by

$$
L_{k}^{\circ}=\hat{\theta}_{1}+\hat{\theta}_{2} \ln \eta_{L_{k}^{\circ}}=\ln \underline{L}_{k}^{\circ},
$$

where $\eta_{L_{k}^{\circ}}$ is a tolerance factor determined by

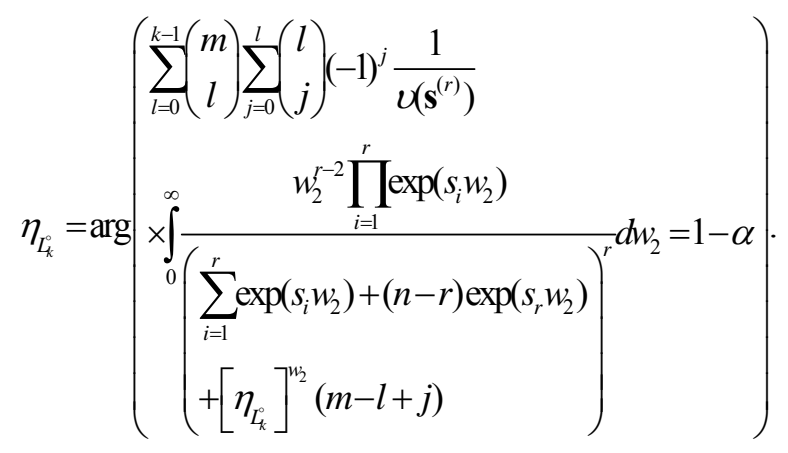

Proof. It follows from (35) and (36) that

$$
\begin{gathered}
\operatorname{Pr}\left(Y_{k}>L_{k}^{\circ}\right)=\int_{L_{k}^{\circ}}^{\infty} g_{\theta}\left(y_{k}\right) d y_{k}=\bar{G}_{\theta}\left(L_{k}^{\circ}\right) \\
=\sum_{l=0}^{k-1}\left(\begin{array}{l}
m \\
l
\end{array}\right) \sum_{j=0}^{l}\left(\begin{array}{l}
l \\
j
\end{array}\right)(-1)^{j}\left[\bar{F}_{\theta}\left(L_{k}^{\circ}\right)\right]^{m-l+j} \\
=\sum_{l=0}^{k-1}\left(\begin{array}{c}
m \\
l
\end{array}\right) \sum_{j=0}^{l}\left(\begin{array}{l}
l \\
j
\end{array}\right)(-1)^{j}\left(\exp \left[-\exp \left(\frac{L_{k}^{\circ}-\theta_{1}}{\theta_{2}}\right)\right]\right)^{m-l+j} \\
=\sum_{l=0}^{k-1}\left(\begin{array}{l}
m \\
l
\end{array}\right) \sum_{j=0}^{l}\left(\begin{array}{l}
l \\
j
\end{array}\right)(-1)^{j}
\end{gathered}
$$

$$
\begin{gathered}
\times\left[\begin{array}{c}
\exp \left[e^{w_{k}}\left(\sum_{i=1}^{r} \exp \left(S_{i} W_{2}\right)+(n-r) \exp \left(S_{r} W_{2}\right)\right)\right. \\
\left.\left.x \exp \left(\frac{L_{k}^{0}-\hat{\theta}}{\hat{\theta}_{2}}\right)\right)^{W_{l}}\left(\sum_{i=1}^{r} \exp \left(S_{i} W_{2}\right)+(n-r) \exp \left(S_{r} W_{2}\right)\right)^{-1}\right]
\end{array}\right)^{m-1+j} \\
=\sum_{l=0}^{k-1}\left(\begin{array}{c}
m \\
l
\end{array}\right) \sum_{j=0}^{l}\left(\begin{array}{l}
l \\
j
\end{array}\right)(-1)^{j}
\end{gathered}
$$$$
\left.\times \exp \left[\begin{array}{l}
-W\left(\exp \left(\frac{L_{k}^{\circ}-\hat{\theta}_{1}}{\hat{\theta}_{2}}\right)\right)^{W_{2}} \\
\times\left(\sum_{i=1}^{r} \exp \left(S_{i} W_{2}\right)+(n-r) \exp \left(S_{r} W_{2}\right)\right)^{-1}(m-l+j)
\end{array}\right]\right) .
$$

Using averaging via pivotal quantity, it follows from (38) and (85) that

$$
E_{\theta}\left\{\operatorname{Pr}\left(Y_{k}>L_{k}^{\circ}(S)\right)\right\}
$$

$$
\begin{aligned}
& \left(\sum_{l=0}^{k-1}\left(\begin{array}{l}
m \\
l
\end{array}\right) \sum_{j=0}^{l}\left(\begin{array}{l}
l \\
j
\end{array}\right)(-1)^{j}\right.
\end{aligned}
$$

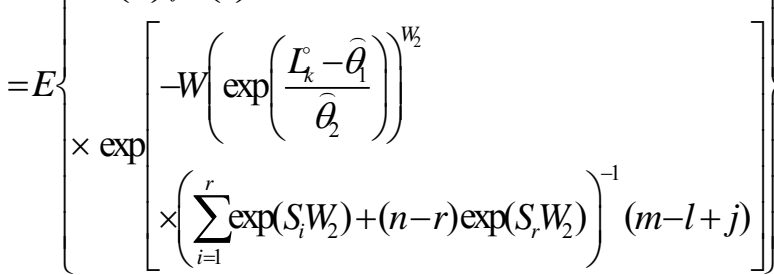

$$
\begin{aligned}
& =\sum_{l=0}^{k-1}\left(\begin{array}{l}
m \\
l
\end{array}\right) \sum_{j=0}^{l}\left(\begin{array}{l}
l \\
j
\end{array}\right)(-1)^{j} \\
& \times \int_{0}^{\infty} \int_{0}^{\infty} \exp \left(\frac{-w\left(\exp \left(\frac{L_{k}^{\circ}-\hat{\theta}_{1}}{\hat{\theta}_{2}}\right)\right)^{w_{2}}(m-l+j)}{\sum_{i=1}^{r} \exp \left(s_{i} w_{2}\right)+(n-r) \exp \left(s_{r} w_{2}\right)}\right) \\
& \times g_{r}(w) f_{n}\left(w_{2} \mid \mathbf{s}^{(r)}\right) d w d w_{2}=\sum_{l=0}^{k-1}\left(\begin{array}{l}
m \\
l
\end{array}\right) \sum_{j=0}^{l}\left(\begin{array}{l}
l \\
j
\end{array}\right)(-1)^{j} \frac{1}{v\left(\mathbf{s}^{(r)}\right)} \\
& \times \int_{0}^{\infty} \frac{w_{2}^{r-2} \prod_{i=1}^{r} \exp \left(s_{i} w_{2}\right)}{\left(\sum_{i=1}^{r} \exp \left(s_{i} w_{2}\right)+(n-r) \exp \left(s_{r} w_{2}\right)\right)^{r}} d w_{2}=1-\alpha .
\end{aligned}
$$


It follows from (86) that

$$
L_{k}^{\circ}=\arg \left(\begin{array}{l}
\sum_{l=0}^{k-1}\left(\begin{array}{l}
m \\
l
\end{array} \sum_{j=0}^{l}\left(\begin{array}{l}
l \\
j
\end{array}\right)^{(-1)^{j} \frac{1}{v\left(\mathbf{s}^{(r)}\right)}} \times \int_{0}^{\infty} \frac{w_{2}^{r-2} \prod_{i=1}^{r} \exp \left(s_{i} w_{2}\right)}{\left(\sum_{i=1}^{r} \exp \left(s_{i} w_{2}\right)+(n-r) \exp \left(s_{r} w_{2}\right)\right)^{r}} d w_{2}=1-\alpha\right. \\
+\left(\exp \left(\frac{L_{k}^{\circ}-\hat{\theta}_{1}}{\hat{\theta}_{2}}\right)\right)^{w_{2}}(m-l+j)
\end{array}\right) .
$$

Assuming that

$$
\exp \left(\frac{L_{k}^{\circ}-\hat{\theta}_{1}}{\hat{\theta}_{2}}\right)=\eta_{L_{k}^{\circ}}
$$

we have (83). This completes the proof.

Theorem 6. Let $\underline{X}_{1} \leq \ldots \leq \underline{X}_{r}$ be the first $r$ ordered observations of a random variable $\underline{X}(=\exp X)$ from a sample of size $n$ from a twoparameter Weibull distribution defined by the probability density function (54). Then a lower statistical tolerance limit with expected $(1-\alpha)$ confidence, $\underline{L}_{k}^{\circ} \equiv \underline{L}_{k}^{\circ}(\underline{S})$, on future outcomes of the $k$ th order statistic $\underline{Y}_{k}\left(=\exp Y_{k}\right)$ from a set of $m$ future ordered observations $\underline{Y}_{1} \leq \ldots \leq \underline{Y}_{m}$ also from the distribution (54), which satisfies (38) is given by

$$
\underline{L}_{k}^{\circ}=\eta_{\underline{L}_{k}^{\circ}}^{1 / \hat{\delta}} \widehat{\beta}=\exp L_{k}^{\circ},
$$

where $\eta_{\underline{L}_{k}^{\circ}}$ is a tolerance factor determined by

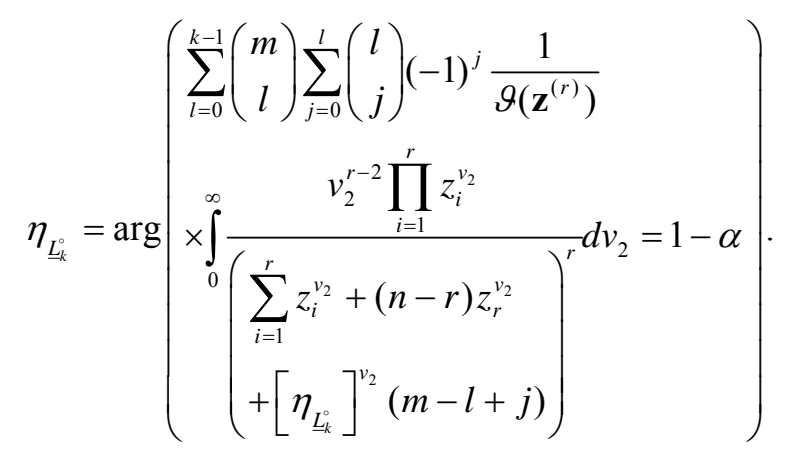

Proof. The proof is similar to that of Theorem 5 and so it is omitted here.
Inference 3 (for the tolerance factors $\eta_{\underline{L}_{k}^{\circ}}$ and $\eta_{L_{k}^{\circ}}$ ). It follows from (88) and (89) that

$$
\eta_{\underline{L}_{k}^{\circ}}=\left(\frac{\underline{L}_{k}^{\circ}}{\widehat{\beta}}\right)^{\hat{\delta}}=\left(\frac{\exp L_{k}^{\circ}}{\exp \hat{\theta}_{1}}\right)^{1 / \hat{\theta}_{2}}=\exp \left(\frac{L_{k}^{\circ}-\hat{\theta}_{1}}{\widehat{\theta}_{2}}\right)=\eta_{L_{k}^{\circ}}
$$

\subsection{Constructing Upper Statistical Tolerance Limit with Expected (1- $\alpha)$ - Confidence}

Theorem 7. Let $X_{1} \leq \ldots \leq X_{r}$ be the first $r$ ordered observations of a random variable $X(=\ln \underline{X})$ from a sample of size $n$ from an extreme-value distribution defined by the probability density function (40). Then an upper statistical tolerance limit with expected $(1-\alpha)$-confidence, $U_{k}^{\circ} \equiv U_{k}^{\circ}(S)$, on future outcomes of the $k$ th order statistic $Y_{k}\left(=\ln \underline{Y}_{k}\right)$ from a set of $m$ future ordered observations $Y_{1} \leq \ldots \leq Y_{m}$ also from the distribution (40), which satisfies (39) is given by

$$
U_{k}^{\circ}=\hat{\theta}_{1}+\hat{\theta}_{2} \ln \eta_{U_{k}^{\circ}}=\ln \underline{U}_{k}^{\circ},
$$

where $\eta_{U_{k}^{\circ}}$ is a tolerance factor determined by

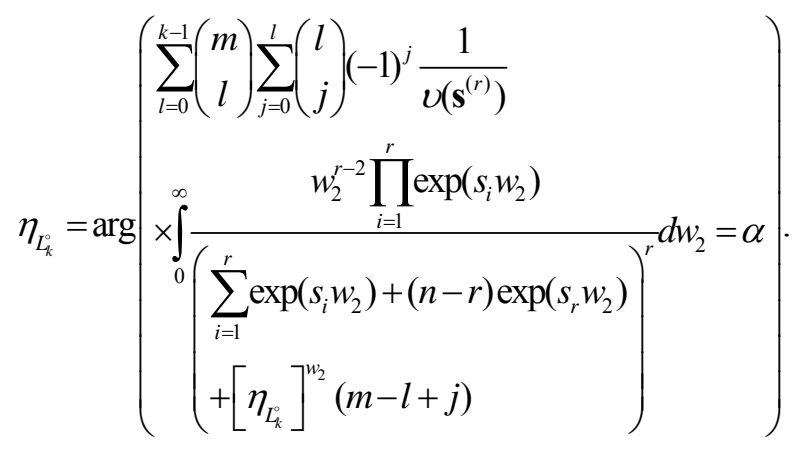

Proof. The upper statistical tolerance limit with expected $\quad(1-\alpha)$-confidence, $\quad U_{k}^{\circ} \equiv U_{k}^{\circ}(S), \quad$ is obtained from a lower statistical tolerance limit with expected $(1-\alpha)$-confidence, $L_{k}^{\circ} \equiv L_{k}^{\circ}(S)$, by replacing $1-\alpha$ by $\alpha$. This completes the proof.

Theorem 8. Let $\underline{X}_{1} \leq \ldots \leq \underline{X}_{r}$ be the first $r$ ordered observations of a random variable $\underline{X}(=\exp X)$ from a sample of size $n$ from a twoparameter Weibull distribution defined by the probability density function (54). Then an upper 
statistical tolerance limit with expected $(1-\alpha)$ confidence, $\underline{U}_{k}^{\circ} \equiv \underline{U}_{k}^{\circ}(\underline{S})$, on future outcomes of the $k$ th order statistic $\underline{Y}_{k}\left(=\exp Y_{k}\right)$ from a set of $m$ future ordered observations $\underline{Y} \leq \ldots \leq \underline{Y}_{m}$ also from the distribution (54), which satisfies (39) is given by

$$
\underline{U}_{k}^{\circ}=\eta_{\underline{U}_{k}^{\circ}}^{1 / \hat{\beta}} \hat{\beta}=\exp U_{k}^{\circ},
$$

where $\eta_{U_{k}^{\circ}}$ is a tolerance factor determined by

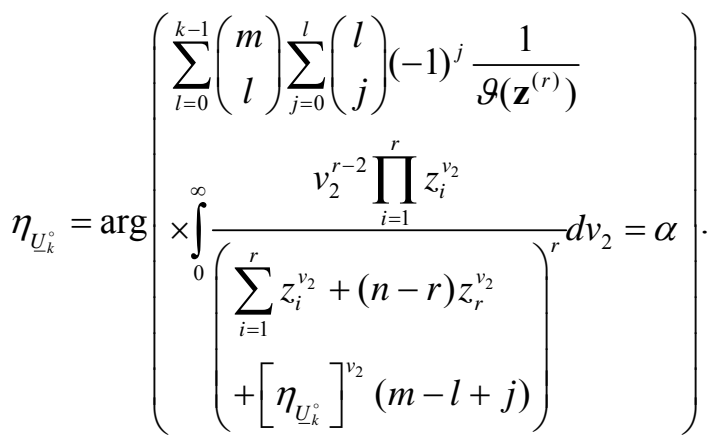

Proof. The upper statistical tolerance limit with expected $\quad(1-\alpha)$-confidence, $\quad \underline{U}_{k}^{\circ} \equiv \underline{U}_{k}^{\circ}(\underline{S}), \quad$ is obtained from a lower statistical tolerance limit with expected $(1-\alpha)$-confidence, $\underline{L}_{k}^{\circ} \equiv \underline{L}_{k}^{\circ}(\underline{S})$, by replacing $1-\alpha$ by $\alpha$. This completes the proof.

Inference 4 (for the tolerance factors $\eta_{\underline{U}_{k}^{\circ}}$ and $\eta_{U_{k}^{\circ}}$ ). It follows from (92) and (94) that

$$
\eta_{\underline{U}_{k}^{\circ}}=\left(\frac{\underline{U}_{k}^{\circ}}{\hat{\beta}}\right)^{\hat{\delta}}=\left(\frac{\exp U_{k}^{\circ}}{\exp \hat{\theta}_{1}}\right)^{1 / \hat{\theta}_{2}}=\exp \left(\frac{U_{k}^{\circ}-\hat{\theta}_{1}}{\hat{\theta}_{2}}\right)=\eta_{U_{k}^{\circ}}
$$

\section{Numerical Examples}

\subsection{Numerical Example 1}

For the Weibull case, Lawless [28] discusses an example with 10 items, which are put on test simultaneously; the life test is terminated at the time of the fifth failure, whence $n=10, r=5$, in our notation here; $\underline{X}_{1}=50.5$ hours, $\underline{X}_{2}=71.3, \underline{X}_{3}=84.6$, $\underline{X}_{4}=98.7, \underline{X}_{5}=103.8$; the maximum likelihood estimates of $\delta$ and $\beta$ are, respectively,

$$
\begin{gathered}
\widehat{\delta}=\left[\begin{array}{l}
\left(\sum_{i=1}^{r} \underline{x}_{i}^{\hat{\delta}} \ln \underline{\underline{X}}_{i}+(n-r) \underline{x}_{r}^{\hat{\delta}} \ln \underline{x}_{r}\right) \\
\times\left(\sum_{i=1}^{r} \underline{x}_{i}^{\hat{\delta}}+(n-r) \underline{x}_{r}^{\hat{\delta}}\right)^{-1}-\frac{1}{r} \sum_{i=1}^{r} \ln \underline{x}_{i}
\end{array}\right]^{-1}=4.199, \\
\widehat{\beta}=\left(r^{-1}\left(\sum_{i=1}^{r} \underline{x}_{i}^{\hat{\delta}}+(n-r) \underline{X}_{r}^{\hat{\delta}}\right)\right)^{1 / \hat{\delta}}=114.2796 .
\end{gathered}
$$

Based on these data, a lower $90 \%$ prediction limit (in terms of this paper, a lower statistical tolerance limit with expected $(1-\alpha)$-confidence, where $\alpha=0.1$ ) is to be constructed for the minimum of 40 independently, identically distributed lifetimes. Lawless [28] reports a conditional lower 90\% prediction limit of 8.8 hours for this example. Based on a simulation of 50000 samples, the lower prediction limit obtained by Mee and Kushary [29] is 8.73 hours.

\section{Lower statistical tolerance limit with expected} (1- $\alpha$ )-confidence. Taking $1-\alpha=0.9$ and $k=1$, with $n=10, r=5$ and $m=40$, we have from (89) that the lower statistical tolerance limit with expected (1- $\alpha)$-confidence, $\underline{L}_{k}^{\circ} \equiv \underline{L}_{k}^{\circ}(\underline{S})$, on the minimum $\left(\underline{Y}_{1}\right)$ of independent lifetimes in a group of $m=40$ components which are to be put into service, is given by

$$
\underline{L}_{k}^{\circ}=\eta_{\underline{L}_{k}^{\prime}}^{1 / \hat{\beta}} \widehat{\beta}=\exp L_{k}^{\circ}=\exp \left(\widehat{\theta}_{1}+\widehat{\theta}_{2} \ln \eta_{L_{k}^{L_{k}}}\right)=8.7941146
$$

where, as it follows from (90) and (91), the tolerance factor $\eta_{\underline{L}_{k}}$ is given by

$$
\begin{gathered}
\eta_{L_{k}}=\arg \left(\frac{1}{\vartheta\left(\mathbf{z}^{(r)}\right)} \int_{0}^{\infty} \frac{V_{2}^{-2} \prod_{i=1}^{r} z_{i}^{v_{2}}}{\left(\sum_{i=1}^{r} Z_{i}^{v_{2}}+(n-r) Z_{r}^{v_{2}}+\left[\eta_{L_{k}}\right]^{v_{2}} m\right)^{r}} d v_{2}=1-\alpha\right) \\
=\eta_{L_{k}^{\circ}}=2.105 / 10^{5}
\end{gathered}
$$

Statistical inference 1. It is easy to see that the conditional lower $90 \%$ prediction limit of 8.8 hours of Lawless [28] on the minimum $\left(\underline{Y}_{1}\right)$ of independent lifetimes in a group of $m=40$ 
components, which are to be put into service, and the lower statistical tolerance limit of 8.7941146 hours with expected 0.9-confidence, which is obtained in this paper by using the proposed technique, are practically the same.

\section{Lower statistical $\gamma$-content tolerance limit} with expected $(1-\alpha)$-confidence. In the above case (if $\gamma=0.9$ ), it follows from (75) that the lower statistical $\gamma$-content tolerance limit with expected (1- $\alpha)$-confidence, $\underline{L}_{k} \equiv \underline{L}_{k}(\underline{S})$, on the minimum $\left(\underline{Y}_{1}\right)$ of independent lifetimes in a group of $m=40$ components which are to be put into service, is given by

$$
\underline{L}_{k}=\eta_{\underline{L}_{k}}^{1 / \hat{\beta}} \widehat{\beta}=\exp L_{k}=\exp \left(\hat{\theta}_{1}+\hat{\theta}_{2} \ln \eta_{L_{k}}\right)=3.7,(101)
$$

where, as it follows from (76) and (77), the tolerance factor $\eta_{\underline{L}_{k}}$ is given by

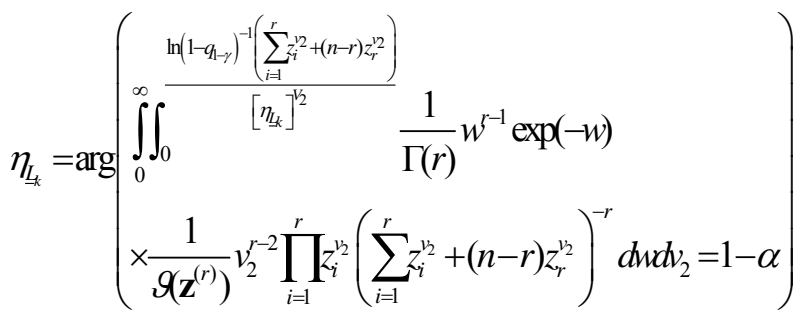

$$
\begin{aligned}
& =\eta_{L_{k}}=5.5451 / 10^{7},
\end{aligned}
$$

$q_{1-\gamma}=0.002631$ is a quantile of the beta distribution satisfying (70).

Statistical inference 2. Thus, the manufacturer has $90 \%$ assurance that no failures will occur in the proportion $\gamma=0.9$ or more of the population of $m=40$ components, which are to be put into service, before $\underline{L}_{k}=3.7$ hours.

Others have computed approximate $90 \%$ prediction limits for a single future lifetime for this example. Fertig, Meyer and Mann [30] computed a lower prediction limit of 56.98 hours, using best linear invariant estimators and a Monte Carlo estimated percentile. Engelhardt and Bain [31] proposed two approximations; for this example they obtained 56.8 (via a procedure requiring iterative solution of a nonlinear equation) and 59.1 (via a simpler approximation). Based on a simulation of 50000 samples, the $90 \%$ lower prediction limit obtained by Mee and Kushary [29] for a single future observation is 56.6 hours.

Lower statistical tolerance limit with expected (1- $\alpha$ )-confidence. Taking $1-\alpha=0.9$ and $k=m=1$, with $n=10$ and $r=5$, we have from (89) that the lower statistical tolerance limit with expected $(1-\alpha)$-confidence, $\underline{L}_{k}^{\circ} \equiv \underline{L}_{k}^{\circ}(\underline{S})$, on a single future observation is given by

$$
\underline{L}_{k}^{\circ}=\eta_{\underline{L}_{k}^{\circ}}^{1 / \hat{\beta}} \widehat{\beta}=\exp L_{k}^{\circ}=\exp \left(\widehat{\theta}_{1}+\hat{\theta}_{2} \ln \eta_{L_{k}^{\circ}}\right)=56.641,
$$

where, as it follows from (90) and (91), the tolerance factor $\eta_{\underline{L}_{k}^{L_{k}}}$ is given by

$$
\begin{gathered}
\eta_{L_{k}^{\circ}}=\arg \left(\frac{1}{\vartheta\left(\mathbf{z}^{(r)}\right)} \int_{0}^{\infty} \frac{v_{2}^{r-2} \prod_{i=1}^{r} z_{i}^{v_{2}}}{\left(\sum_{i=1}^{r} z_{i}^{v_{2}}+(n-r) Z_{r}^{v_{2}}+\left[\eta_{L_{k}^{\circ}}\right]^{v_{2}}\right)^{r}} d v_{2}=1-\alpha\right) \\
=\eta_{L_{k}^{\circ}}=0.052479 .
\end{gathered}
$$

Statistical inference 3. Based on a simulation of 50000 samples, the $90 \%$ lower prediction limit obtained by Mee and Kushary [29] for a single future lifetime is 56.6 hours, which is slightly smaller than 56.641 hours (see (103)). Engelhardt and Bain [31] proposed the first approximate lower prediction limit of 56.8 hours and Fertig, Meyer and Mann [30] computed the lower prediction limit of 56.98 hours for a single future lifetime, which are slightly larger than 56.641 hours (see (103)). The second approximate lower prediction limit of 59.1 hours proposed by Engelhardt and Bain [31] for a single future lifetime is larger than 56.641 hours (see (103)).

\subsection{Numerical Example 2}

Consider the following results given by Lieblein and Zelen [32] of test of endurance, in millions of revolutions, of $n=23$ ball bearings: 17.88, 28.92, $33.00,41.52,42.12,45.60,48.48,51.84,51.96$, $54.12,55.56,67.80,68.64,68.64,68.88,84.12$, $93.12,98.64,105.12,105.84,127.92,128.04$, 
173.40. The maximum likelihood estimates of $\delta$ and $\beta$ are, respectively,

$$
\begin{gathered}
\widehat{\delta}=\left[\left(\sum_{i=1}^{n} \underline{\underline{x}}_{i}^{\hat{\delta}} \ln \underline{\underline{x}}_{i}\right)\left(\sum_{i=1}^{n} \underline{\underline{x}}_{i}^{\hat{\delta}}\right)^{-1}-\frac{1}{n} \sum_{i=1}^{n} \ln \underline{\underline{x}}_{i}\right]^{-1}=2.102, \\
\widehat{\beta}=\left(n^{-1}\left(\sum_{i=1}^{n} \underline{\underline{x}}_{i}^{\hat{\delta}}\right)\right)^{1 / \hat{\delta}}=81.878
\end{gathered}
$$

Numerous authors have used these data as illustrative of a sample from a two-parameter Weibull distribution. Using 20000 simulated samples of size $n=23$, Mee and Kushary [29] obtained a $90 \%$ lower prediction limit for the fifth failure out one hundred ball bearings equal to 10.11 million of revolutions, which is slightly smaller than the two approximate prediction limits 10.27 and 10.59 reported by Engelhardt and Bain [31].

\section{Lower statistical tolerance limit with expected} (1- $\alpha$ )-confidence. Taking $1-\alpha=0.9$ and $k=5$, with $r=n=23$ and $m=100$, we have from (89) that the lower statistical tolerance limit with expected (1- $\alpha)$-confidence, $\underline{L}_{k}^{\circ} \equiv \underline{L}_{k}^{\circ}(\underline{S})$, for a fifth failure out one hundred ball bearings is given by

$$
\underline{L}_{k}^{\circ}=\eta_{\underline{L}_{k}^{\circ}}^{1 / \hat{\beta}} \widehat{\beta}=\exp L_{k}^{\circ}=\exp \left(\hat{\theta}_{1}+\widehat{\theta}_{2} \ln \eta_{L_{k}^{\circ}}\right)=10.35206,
$$

where, as it follows from (90) and (91), the tolerance factor $\eta_{\underline{L}_{k}^{\circ}}$ is given by

$$
\eta_{\underline{L}_{k}^{\circ}}=\arg \left(\begin{array}{c}
\sum_{l=0}^{k-1}\left(\begin{array}{l}
m \\
l
\end{array}\right) \sum_{j=0}^{l}\left(\begin{array}{l}
l \\
j
\end{array}\right)(-1)^{j} \frac{1}{\vartheta\left(\mathbf{z}^{(n)}\right)} \\
\times \int_{0}^{\infty} \frac{v_{2}^{n-2} \prod_{i=1}^{n} z_{i}^{v_{2}}}{\left(\sum_{i=1}^{n} z_{i}^{v_{2}}+\left[\eta_{L_{k}^{\circ}}\right]^{v_{2}}(m-l+j)\right)^{n}} d v_{2}=1-\alpha \\
=\eta_{L_{k}^{\circ}}=0.0129452 .
\end{array}\right.
$$

Statistical inference 4. Thus, it follows from (107) that the lower statistical tolerance limit with expected confidence of $0.9 \quad\left(\underline{L}_{k}^{\circ}=10.35206\right.$ million of revolutions) is between the two approximate prediction limits 10.27 and 10.59 reported by
Engelhardt and Bain [31].

Lower statistical tolerance limit with expected (1- $\alpha$ )-confidence. Taking $1-\alpha=0.9$ and $k=1$, with $r=n=23$ and $m=100$, we have from (89) that the lower statistical tolerance limit with expected (1- $\alpha)$-confidence, $\underline{L}_{k}^{\circ} \equiv \underline{L}_{k}^{\circ}(\underline{S})$, for a first failure out one hundred ball bearings, is given by

$$
\underline{L}_{k}^{\circ}=\eta_{\underline{L}_{k}^{\circ}}^{1 / \widehat{\delta}} \widehat{\beta}=\exp L_{k}^{\circ}=\exp \left(\hat{\theta}_{1}+\hat{\theta}_{2} \ln \eta_{L_{k}^{\circ}}\right)=2.083,
$$

where, as it follows from (90) and (91), the tolerance factor $\eta_{\underline{L}_{k}}$ is given by

$$
\begin{gathered}
\eta_{\underline{L}_{k}^{\circ}}=\arg \left(\frac{1}{\vartheta\left(\mathbf{z}^{(n)}\right)} \int_{0}^{\infty} \frac{v_{2}^{n-2} \prod_{i=1}^{n} z_{i}^{v_{2}}}{\left(\sum_{i=1}^{n} z_{i}^{v_{2}}+\left[\eta_{L_{k}^{\circ}}\right]^{v_{2}} m\right)^{n}} d v_{2}=1-\alpha\right) \\
=\eta_{L_{k}^{\circ}}=0.00044503 .
\end{gathered}
$$

Statistical inference 5. Lawless [17] obtained for this example (via conditional approach in terms of a extreme-value (Gumbel) distribution) the lower $90 \%$ prediction limit of 2.08 million of revolutions for a first failure out one hundred ball bearings, which is slightly smaller than 2.083 (see (109)).

\subsection{Numerical Example 3}

Consider the data in an example discussed by Mann and Saunders [33]. They regard the data coming from the Weibull distribution as the results of full scale fatigue tests on a particular type of component. The data are for a complete sample of size $n=3$, with observations $\underline{X}_{1}=45.952, \underline{X}_{2}=54.143$, and $X_{3}=65.440$, results being expressed here in number of thousands of cycles. On the basis of these data it is wished to obtain the lower statistical tolerance limit with expected $(1-\alpha)$-confidence for the minimum $\left(\underline{Y}_{1}\right)$ of independent lifetimes in a group of $m=500$ components which are to be put into service.

\section{Lower statistical tolerance limit with expected} (1- $\alpha)$ confidence. The maximum likelihood 
estimates of the unknown parameters $\delta$ and $\beta$, computed on the basis of $\left(\underline{X}_{1}, \underline{X}_{2}, \underline{X}_{3}\right)$, are $\widehat{\delta}=7.726$ and $\widehat{\beta}=58.706$, respectively. Taking $1-\alpha=0.8$ and $k=1$, with $r=n=3$ and $m=500$, we have from (89) that the lower statistical tolerance limit with expected $(1-\alpha)$-confidence, $\underline{L}_{k}^{\circ} \equiv \underline{L}_{k}^{\circ}(\underline{S})$, on the minimum $\left(\underline{Y}_{1}\right)$ of independent lifetimes in a group of $m=500$ components which are to be put into service, is given by

$$
\underline{L}_{k}^{\circ}=\eta_{\underline{L}_{k}^{\circ}}^{1 / \hat{\beta}} \hat{\beta}=\exp L_{k}^{\circ}=\exp \left(\hat{\theta}_{1}+\hat{\theta}_{2} \ln \eta_{L_{k}^{\circ}}\right)=5.527411,(111)
$$

where, as it follows from (90) and (91), the tolerance factor $\eta_{\underline{\underline{L}}_{k}^{\circ}}$ is given by

$$
\begin{gathered}
\eta_{\underline{L}_{k}^{\circ}}=\arg \left(\frac{1}{\vartheta\left(\mathbf{z}^{(n)}\right)} \int_{0}^{\infty} \frac{v_{2}^{n-2} \prod_{i=1}^{n} z_{i}^{v_{2}}}{\left(\sum_{i=1}^{n} z_{i}^{v_{2}}+\left[\eta_{L_{k}^{\circ}}\right]^{v_{2}} m\right)^{n}} d v_{2}=1-\alpha\right) \\
=\eta_{L_{k}^{\circ}}=1.18 / 10^{8} .
\end{gathered}
$$

Statistical inference 6. Lawless [28] obtained for this example (via conditional approach in terms of the extreme-value (Gumbel) distribution) the lower $80 \%$ prediction limit of 5.623 , which is slightly larger than 5.527411 (see (111)). The resulting lower $80 \%$ prediction limit of Mee and Kushary [29] for this example (obtained via simulation of 100000 samples) was 5.225, which is slightly smaller than 5.527411 (see (111)). The Mann and Saunders [33] result for this example was only 0.766 . All results are expressed here in the number of thousands of cycles.

Lower statistical $\gamma$-content tolerance limit with expected $(\mathbf{1}-\boldsymbol{\alpha})$-confidence. Taking $\gamma=0.8$, $1-\alpha=0.8$ and $k=1$, with $r=n=3$ and $m=500$, we have from (75) that a lower statistical $\gamma$-content tolerance limit with expected $(1-\alpha)$-confidence, $\underline{L}_{k} \equiv \underline{L}_{k}(\underline{S})$, on the minimum $\left(\underline{Y}_{1}\right)$ of independent lifetimes in a group of $m=500$ components which are to be put into service, is

$$
\underline{L}_{k}=\eta_{\underline{L}_{k}}^{1 / \hat{\beta}} \hat{\beta}=\exp L_{k}=\exp \left(\hat{\theta}_{1}+\hat{\theta}_{2} \ln \eta_{L_{k}}\right)=4.082282
$$

where, as it follows from (76) and (77), the tolerance factor $\eta_{\underline{L}_{k}}$ is given by

$$
\eta_{L_{k}}=\arg \left(\begin{array}{c}
\int_{0}^{\infty} \int_{0}^{\frac{\ln \left(1-q_{1-\gamma}\right)}{\left[\eta_{L_{k}}\right]^{-1} \sum_{i=1}^{n} Z_{i}^{\eta^{2}}}} \frac{1}{\Gamma(n)} w^{n-1} \exp (-w) \\
\times \frac{1}{\vartheta\left(\mathbf{z}^{(n)}\right)} v_{2}^{n-2} \prod_{i=1}^{n} z_{i}^{v_{2}}\left(\sum_{i=1}^{n} z_{i}^{v_{2}}\right)^{-n} d w d v_{2}=1-\alpha
\end{array}\right)
$$

$q_{1-\gamma}$ is a quantile of the beta distribution satisfying (70).

Statistical inference 7. Thus, the manufacturer has $80 \%$ assurance that no failures will occur in the proportion $\gamma=0.8$ or more of the population of $m=500$ components, which are to be put into service, before $\underline{L}_{k}=4.082282$ thousands of cycles.

\section{Future Research Directions}

Predictive inferences (via tolerance limits) for future outcomes on the basis of the past and present knowledge represent a fundamental problem of statistics, arising in many contexts and producing varied solutions. In this paper, new-sample prediction (via tolerance limits) based on a previous sample is considered (i.e., when for predicting the future outcomes in a new sample there are available the observed data only from a previous sample). It is interesting to consider within-sample prediction (via tolerance limits) based on the early data from a current experiment (i.e., when for predicting the future outcomes in a sample there are available the early data only from that sample), and new-withinsample prediction (via tolerance limits) based on both the early data from that sample and the data from a previous sample (i.e., when for predicting the future outcomes in a new sample there are available both the early data from that sample and the data from a previous sample), where it is assumed that only the functional form of the underlying distributions is specified, but some or all of its parameters are unspecified.

\section{Conclusion}


In this article, we construct the following one-sided statistical tolerance limits: i) one-sided statistical tolerance limit that covers at least $100 \gamma \%$ of the measurements with expected $100(1-\alpha) \%$ confidence, ii) one-sided statistical tolerance limit determined so that the expected proportion of the measurements covered by this limit is $(1-\alpha)$. Tolerance limits have important role in application of statistical methods in technical practice, especially in statistical quality control. Inherent in every phase of industrial quality control is the problem of comparing some quality characteristic or measurement of a finished product against given specifications. Sometimes the specifications, or tolerance limits, are so stated by the customer or by design engineer that any appreciable departure will make the product unusable. There remains, however, the problem of producing the part so that an acceptably high proportion of units will fall within tolerance limits specified for the given quality characteristic. Also, if a product is made without prior specifications, or if modifications are made, it is desirable to know within what limits the process can hold a quality characteristics a reasonably high percentage of the time. We thus speak of natural tolerance limits; that is, we let the process establish its own limits which, according the experience, can be met in actual practice. The new analytical technique proposed in this article represents the conceptually simple, efficient and useful method for constructing exact statistical tolerance limits on future outcomes under parametric uncertainty of underlying models. It does not in need to make any assumption concerning the statistical equation for the tolerance limit. This technique, using the experimental complete or type II censored data, is based on the idea of invariant embedding of a sample statistic in the underlying model to construct pivotal quantities and to eliminate the unknown parameters from the problem via pivotal quantity averaging. In this case, the exact statistical tolerance limits (under parametric uncertainty of underlying models) on future outcomes (say, order statistics) associated with sampling from corresponding distributions can be found easily and quickly making tables, simulation and special computer programs unnecessary.

The analytical methodology described in this paper is illustrated for the two-parameter Weibull and extreme-value distributions. Applications to other log-location-scale distributions could follow directly.
Finally, we give the three illustrative numerical examples, where the exact statistical tolerance limits with expected $(1-\alpha)$-confidence, obtained in this paper in terms of the two-parameter Weibull or extreme-value distribution, are compared with the known results that are reported in the literature and were obtained by using the following: 1) tables, 2) simulation, 3) Monte Carlo estimated percentiles, 4) special computer programs, 5) approximation, 6) transformation of the two-parameter Weibull distribution to the extreme-value distribution, etc. Although the details of the problems addressed in the paper can vary significantly from one industry to another, the focus is always on making more accurate decisions, rather than manually using guesses and intuitions, but rather from a scientific point of view using models and technologies implemented with disciplined processes and systems.The methodologies described here can be extended in several different directions to solve various problems arising in practice.

\section{References:}

[1] Berk, R.H., A special group structure and equivariant estimation, Ann. Math. Statist., Vol. 38, 1967, pp. 1436-1446.

[2] Ferguson, T.S., Mathematical Statistics, New York: Academic Press, 1967.

[3] Patel, J.K., Tolerance limits: a review, Communications in Statistics: Theory and Methodology, Vol. 15, 1986, pp. 2719-2762.

[4] Dunsmore, J.R., Some approximations for tolerance factors for the two parameter exponential distribution, Technometrics, Vol. 20, 1978, pp. 317-318.

[5] Guenther, W.C., Patil, S.A., and Uppuluri, V.R.R., One-sided $\beta$-content tolerance factors for the two parameter exponential distribution, Technometrics, Vol. 18, 1976, pp. 333-340.

[6] Engelhardt, M. and Bain, L.J., Tolerance limits and confidence limits on reliability for the two-parameter exponential distribution, Technometrics, Vol. 20, 1978, pp. 37-39.

[7] Guenther, W.C., Tolerance intervals for univariate distributions, Naval Research Logistics Quarterly, Vol. 19, 1972, pp. 309333. 
[8] Hahn, G.J. and Meeker, W.Q., Statistical Intervals: A Guide for Practitioners, New York: John Wiley \& Sons, 1991.

[9] Nechval, K.N. and Nechval, N.A.,Constructing lower simultaneous prediction limits on observations in future samples from the past data, Computers \& Industrial Engineering, Vol. 137, 1999, pp. 133-136.

[10] Nechval, N.A. and Vasermanis, E.K., Improved Decisions in Statistics, Riga: Izglitibas soli, 2004.

[11] Nechval, N.A., Berzins, G., Purgailis, M., and Nechval, K.N., Improved estimation of state of stochastic systems via invariant embedding technique, WSEAS Transactions on Mathematics, Vol. 7, 2008, pp. 141-159.

[12] Nechval, N.A., Nechval, K.N., Berzins, G., Purgailis, M., and Rozevskis, U., Stochastic fatigue models for efficient planning inspections in service of aircraft structures, in Analytical and Stochastic Modeling Techniques and Applications, Al-Begain, K., Heindl, A., and Telek, M., Eds., Berlin: Springer-Verlag, Heidelberg, LNCS, Vol. 5055, 2008, pp. 114-127.

[13] Nechval, N.A., Nechval, K.N., Danovich V., and Liepins, T., Optimization of new-sample and within-sample prediction intervals for order statistics, in Proceedings of the 2011 World Congress in Computer Science, Computer Engineering, and Applied Computing, WORLDCOMP'11, Las Vegas, Nevada, USA, 18-21 July, 2011, pp. 91-97.

[14] Nechval, K.N. and Nechval, N.A., Technique of constructing predictive inferences for future outcomes with applications to inventory management problems, Adv. Syst. Sci. Appl., Vol. 13, 2013, pp. 355-368.

[15] Nechval, N.A. and Nechval, K.N., A new approach to constructing simultaneous prediction limits on future outcomes under parametric uncertainty of underlying models, in IAENG Transactions on Engineering Sciences, Ao Sio-long, Chan Alan Hoi-Shou, Katagiri Hideki, and $\mathrm{Xu} \mathrm{Li}$, Eds., London: Taylor \& Francis Group, 2014, pp. 1-13.

[16] Nechval, N.A., Nechval, K.N., Danovich, V., and Berzins, G., Predictive inferences for future order statistics coming from an inverse Gaussian distribution, in Lecture Notes in Engineering and Computer Science: Proceedings of The World Congress on Engineering, London, U.K., 2-4 July, 2014, pp. $888-893$.

[17] Nechval, N.A., Nechval, K.N., and Berzins, G., A new technique for intelligent constructing exact $\gamma$-content tolerance limits with expected $(1-\alpha)$-confidence on future outcomes in the Weibull case using complete or Type II censored data, Automatic Control and Computer Sciences (AC \& CS), Vol. 52, 2014, pp. 476-488.

[18] Nechval, N.A. and Nechval, K.N., Improved planning in-service inspections of fatigued aircraft structures under parametric uncertainty of underlying lifetime models, in Numerical Methods for Reliability and Safety Assessment: S. Kadry and A. El. Hami, Eds., Springer, Switzerland, 2015, pp. 647-674.

[19] Nechval, N.A. and Nechval, K.N., Tolerance limits on order statistics in future samples coming from the two-parameter exponential distribution, American Journal of Theoretical and Applied Statistics (AJTAS), Vol. 5, 2016, pp. 1-6.

[20] Nechval, N.A., Nechval, K.N., Prisyazhnyuk, S.P., and Strelchonok, V.F., Tolerance limits on order statistics in future samples coming from the Pareto distribution, Automatic Control and Computer Sciences (AC \& CS), Vol. 50, 2016, pp. 423-431.

[21] Nechval,N.A.,Nechval, K.N., and Strelchonok, V.F.,A new approach to constructing tolerance limits on order statistics in future samples coming from a normal distribution, Advances in Image and Video Processing (AIVP), Vol. 4, 2016, pp. 47-61.

[22] Nechval, N.A., Berzins, G., Balina, G.S., Steinbuka, I., and Nechval, K.N., Constructing unbiased prediction limits on future outcomes under parametric uncertainty of underlying models via pivotal quantity averaging approach, Automatic Control and Computer Sciences (AC \& CS), Vol. 51, 2017, pp. 331346.

[23] Nechval, N.A., Nechval, K.N., and Berzins, G., A new technique for constructing exact tolerance limits on future outcomes under 
parametric uncertainty, in Advanced Mathematical Techniques in Engineering Sciences, M. Ram and J. P. Davim, Eds. London: Taylor \& Francis Group, 2018, pp. 203-226.

[24] Nechval, N.A., Berzins, G., and Nechval, K.N., Intelligent planning reliability-based inspections of fatigued structures for the crack initiation period in the Weibull case under parametric uncertainty, Automatic Control and Computer Sciences (AC \& CS), Vol. 52, 2018, pp. 184-197.

[25] Nechval, N.A., Berzins, G., Nechval, K.N., and Krasts, J., A new technique of intelligent constructing unbiased prediction limits on future order statistics coming from an inverse Gaussian distribution under parametric uncertainty, Automatic Control and Computer Sciences (AC \& CS), Vol. 53, 2019, pp. 223235.

[26] Nechval, N.A., Berzins, G., and Nechval, K.N., Intelligent technique of constructing exact statistical tolerance limits to predict future outcomes under parametric uncertainty for prognostics and health management of complex systems, International Journal of Advances in Computer Science \& Its Applications (JCSIA), Vol. 9, No. 2, 2019, pp. 30-47.

[27] Gumbel, E.J., Statistics of Extreme, New York: Columbia University Press, 1958.

[28] Lawless, J.F., On the estimation of safe life when the underlying life distribution is Weibull, Technometrics, Vol. 15, 1973, pp. 857-865.

[29] Mee, R.W. and Kushary, D., Prediction limits for the Weibull distribution utilizing simulation, Computational Statistics \& Data Analysis, Vol. 17, 1994, pp. 327-336.

[30] Fertig, K.W., Meyer, M.E., and Mann, N.R., On constructing prediction intervals from a Weibull or extreme-value distribution, Technometrics, Vol. 22, 1980, pp. 567-573.

[31] Engelhardt, M. and Bain, L.J., On prediction limits for samples from a Weibull or extremevalue distribution, Technometrics, Vol. 24, 1982, pp. 147-150.
[32] Lieblein, J. and Zelen, M., Statistical investigation of the fatigue life of deep-groove ball bearing, Journal of Research of the National Bureau of Standards, Vol. 47, 1956, pp. 273-316.

[33] Mann, N.R. and Saunders, S.C., On evaluation of warranty assurance when life has a Weibull distribution, Biometrika, Vol. 56, 1969, pp. 615-625.

[34] Bsili Ibtissem, Ghabi Jalel, Hassani Messaoud, Discrete Time Sliding Mode Control of Nonlinear Uncertain Systems Based on Estimation of Uncertainties and External Disturbances, WSEAS Transactions on Systems and Control, Volume 13, 2018, Art. \#9, pp. 74-79 